\title{
Membralin deficiency dysregulates astrocytic glutamate homeostasis, leading to ALS-like impairment
}

\author{
Lu-Lin Jiang, ${ }^{1}$ Bing Zhu, ${ }^{1}$ Yingjun Zhao, ${ }^{1}$ Xiaoguang Li, ${ }^{1}$ Tongfei Liu, ${ }^{1}$ Juan Pina-Crespo, ${ }^{1}$ Lisa Zhou, ${ }^{1}$ Wenxi Xu, ${ }^{2}$ \\ Maria J. Rodriguez, ${ }^{3,4}$ Haiyang Yu, ${ }^{5}$ Don W. Cleveland, , ${ }^{3,5,6}$ John Ravits, ${ }^{3,4}$ Sandrine Da Cruz, ${ }^{5}$ Tao Long, ${ }^{2}$ Dongxian Zhang, \\ Timothy Y. Huang, ${ }^{1}$ and Huaxi $X \mathbf{u}^{1}$
}

1'Neuroscience Initiative, Sanford Burnham Prebys Medical Discovery Institute, La Jolla, California, USA. Bioinformatics and Structural Biology Program, Sanford Burnham Prebys Medical Discovery Institute, La Jolla, California, USA. ${ }^{3}$ Department of Neurosciences, UCSD, La Jolla, California, USA. ${ }^{4}$ ALS Translational Research Program, Department of Neurosciences, UCSD, La Jolla, California, USA. ${ }^{5}$ Ludwig Institute for Cancer Research, UCSD, La Jolla, California, USA. ${ }^{6}$ Department of Cellular and Molecular Medicine, UCSD, La Jolla, California, USA.

\begin{abstract}
Mechanisms underlying motor neuron degeneration in amyotrophic lateral sclerosis (ALS) are yet unclear. Specific deletion of the ER-component membralin in astrocytes manifested postnatal motor defects and lethality in mice, causing the accumulation of extracellular glutamate through reducing the glutamate transporter EAAT2. Restoring EAAT2 levels in membralin-KO astrocytes limited astrocyte-dependent excitotoxicity in motor neurons. Transcriptomic profiles from mouse astrocytic membralin-KO motor cortex indicated significant perturbation in KECG pathway components related to ALS, including downregulation of Eaat2 and upregulation of Tnfrsf1a. Changes in gene expression with membralin deletion also overlapped with mouse ALS models and reactive astrocytes. Our results show that activation of the TNF receptor (TNFR1) NFKB pathway known to suppress Eaat2 transcription was upregulated with membralin deletion. Further, reduced membralin and EAAT2 levels correlated with disease progression in spinal cord from SOD1-mutant mouse models, and reductions in membralin/EAAT2 were observed in human ALS spinal cord. Importantly, overexpression of membralin in SOD1C93A astrocytes decreased TNFR1 levels and increased EAAT2 expression, and improved motor neuron survival. Importantly, upregulation of membralin in $S O D 1^{C 93 A}$ mice significantly prolonged mouse survival. Our study provided a mechanism for ALS pathogenesis where membralin limited glutamatergic neurotoxicity, suggesting that modulating membralin had potential in ALS therapy.
\end{abstract}

\section{Introduction}

Glutamate is an essential excitatory neurotransmitter and, at concentrations of approximately $6 \mu \mathrm{mol} / \mathrm{g}$ (wet weight) in human brain, is the most abundant free amino acid species in the central nervous system $(1,2)$. Release of presynaptic glutamate results in stimulation of ionotropic and metabotropic glutamate receptors at postsynaptic densities, which requires tight regulation of extrasynaptic glutamate levels and glutamate within the synaptic cleft (3). Excessive glutamate overactivates ionotropic glutamate receptors, which deteriorates postsynaptic spines, dendrites, and neuronal cell bodies (4). Homeostatic maintenance of extracellular glutamate levels is primarily mediated by astrocytic cell-surface glutamate transporters. Reduction of glial glutamate transporters (EAAT1, EAAT2) in mice produced marked effects in elevating extracellular glutamate levels, whereas little or no change in extracellular glutamate is observed with deletion of the neuronal transporter (EAAT3/EAAC1) (5).

Authorship note: $B Z, Y Z$, and $X L$ contributed equally to this work. DZ is deceased. Conflict of interest: The authors have declared that no conflict of interest exists. Copyright: (c) 2019, American Society for Clinical Investigation.

Submitted: January 24, 2019; Accepted: May 10, 2019; Published: June 24, 2019

Reference information: / Clin Invest. 2019;129(8):3103-3120.

https://doi.org/10.1172/JCl127695.
Consequent effects of deleting astrocytic glutamate transporters in mice implicate the importance of extracellular glutamate homeostasis in physiological brain function. Hemizygous deletion of EAAT2 features behavioral defects indicative of mild glutaminergic overstimulation (6), whereas homozygous EAAT2 deletion results in spontaneous seizures at 6 weeks of age, with a high rate of lethality (7). Additionally, deletion of EAAT1 in mice has been shown to impair adaptation to advanced motor coordination (8). As this implicates the importance of tight glutamate regulation in normal brain function, a pathophysiological role for glutamate excitotoxicity and glutamate transporter impairment has been established in various neurological disorders. Glutamate excitotoxicity has been hypothesized to play a role in motor neuron death in amyotrophic lateral sclerosis (ALS) (9-11), and motor neuron hyperexcitability is also apparent in presymptomatic $S O D 1^{G 93 A}$ ALS mouse models (12). Interestingly, marked reduction of EAAT2 has been observed in spinal cord from patients with ALS, correlating with regions of motor neuron loss (13). Decreased EAAT2 immunoreactivity is also seen to be most pronounced in pathologically affected regions such as the spinal cord $(14,15)$, further implicating a role for EAAT2 dysregulation in motor neuron degeneration. Indeed, impaired EAAT2 expression has been observed in transgenic ALS SOD1 ${ }^{G 93 A}$ mouse $(16,17)$ and rat $(18)$ models, as well as TDP43 ALS mouse models (19). Although how 
A
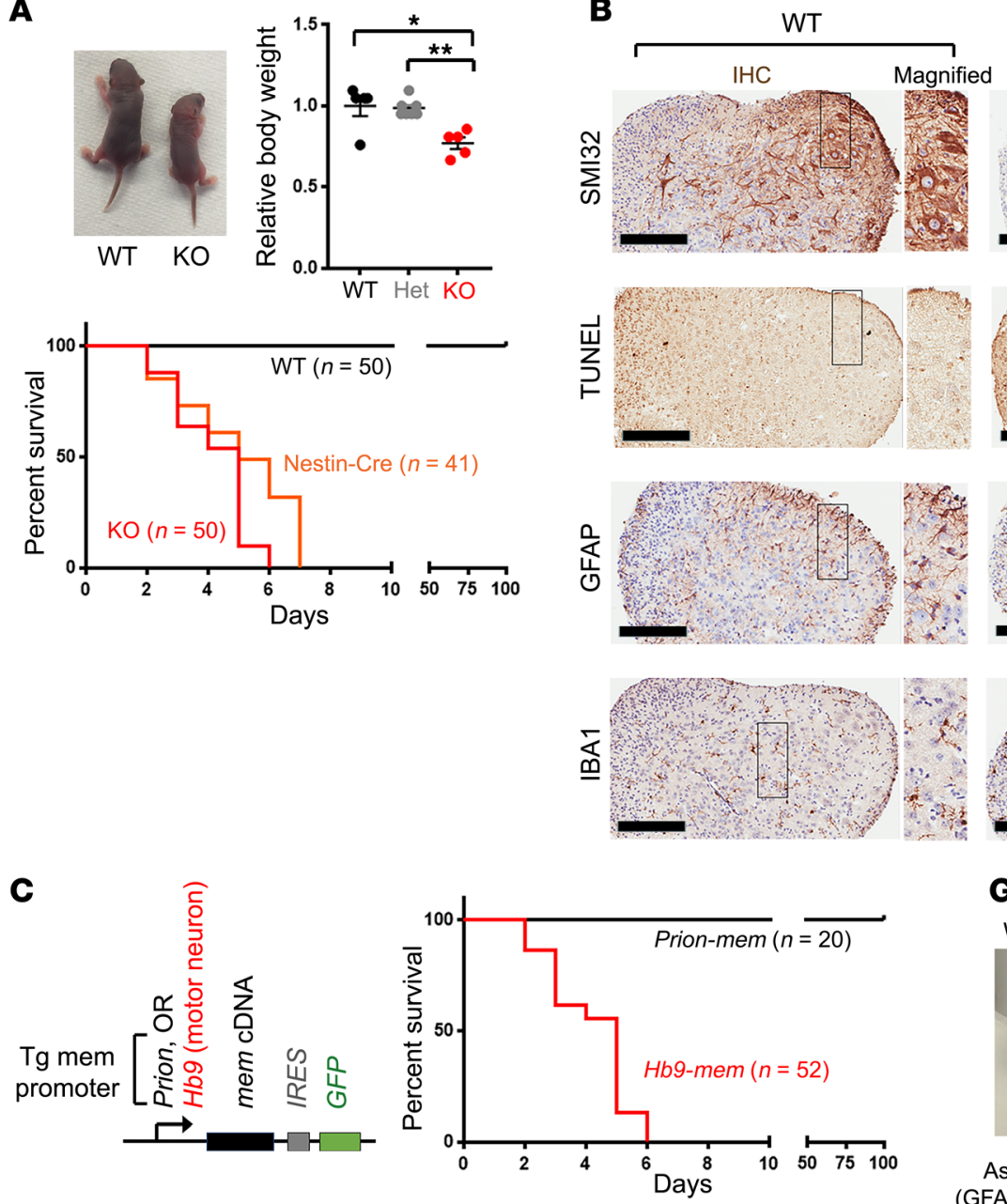

D

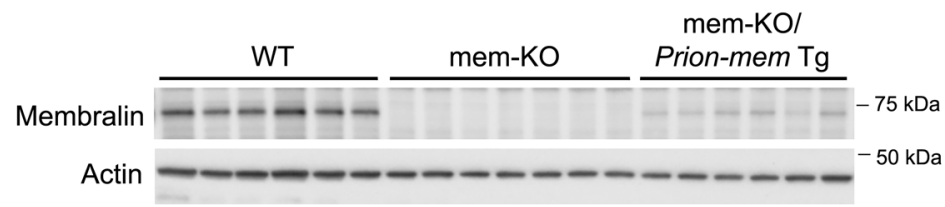

$\mathbf{E}$
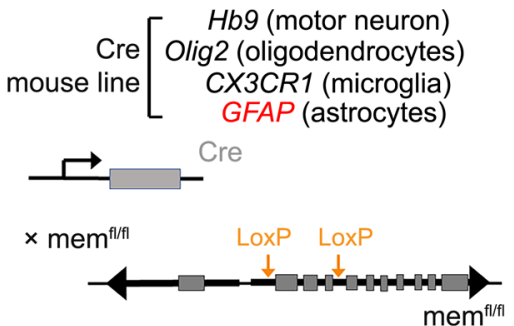

$\mathbf{F}$

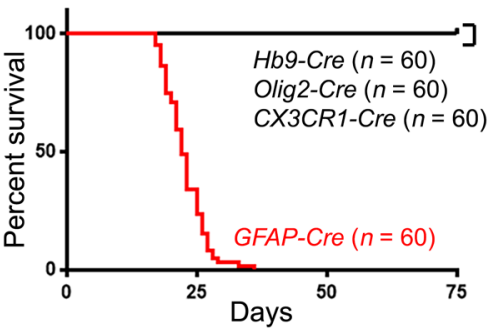

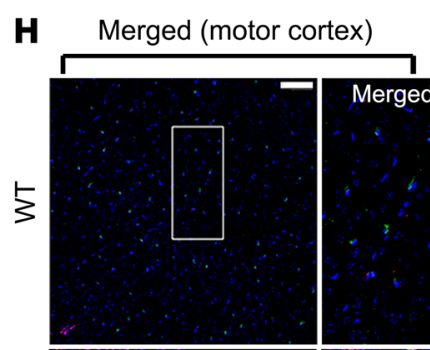
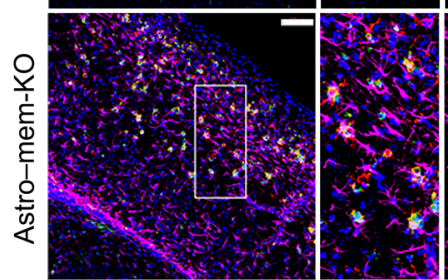


\section{G}

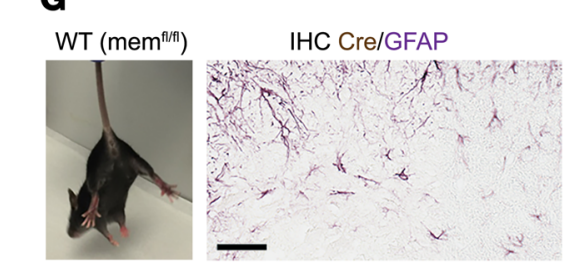

Astro-mem-KO

$\left(\right.$ GFAP-Cre $\times$ mem $^{\mathrm{fl} / \mathrm{fl}}$ ) IHC Cre/GFAP
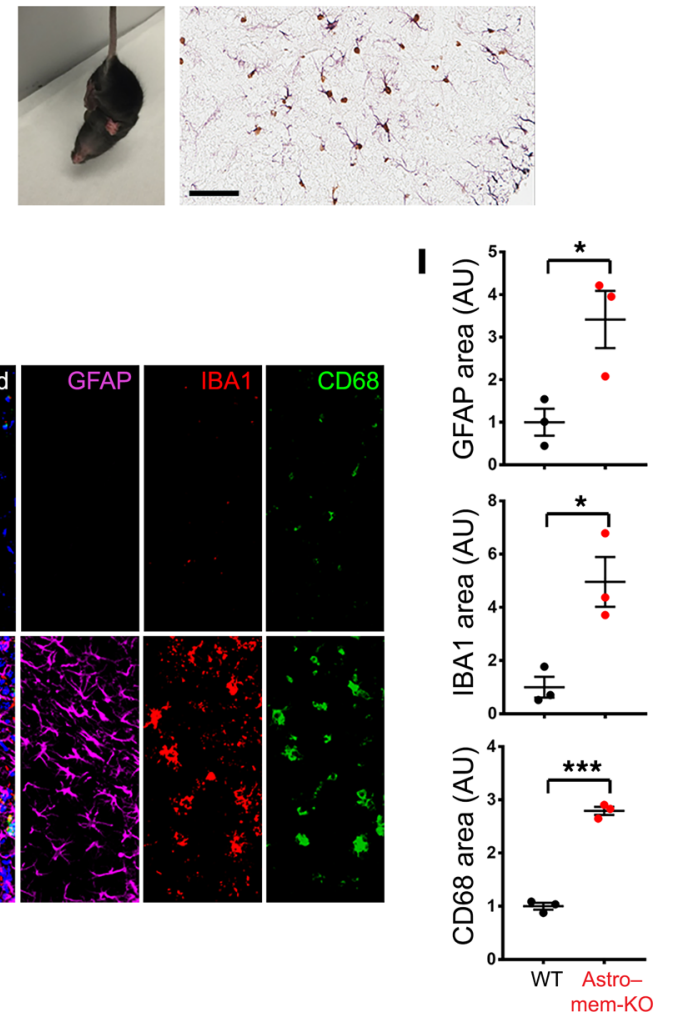





Figure 1. Membralin deletion in astrocytes manifests motor defects and early postnatal lethality. (A) WT and membralin whole-body deletion (mem-KO) animals at P3 (reshown in Supplemental Figure 1B). Relative body weight of WT (black), mem heterozygous (gray), and homozygous KO (red) animals at P3 (WT animals set to 1.0). Bottom graph shows Kaplan-Meier survival curves for WT (black), whole-body mem-KO (red), and Nestin-Cre mem-KO (Nestin-Cre) animals (orange). (B) SMI32 (motor neurofilaments), TUNEL (apoptosis), GFAP (astrocytes), and IBA1 (microglia) staining in spinal cord from P3 WT and KO animals. Scale bars: 200 $\mu \mathrm{m}$. Graphs indicate quantified motor neuron number, cell death, and astroglial/microglial staining area (mean \pm SE). GFAP and IBA1 staining was compared with WT (normalized to 1.0 AU). (C) Schematic of transgenic membralin Tg overexpression mouse lines under Prion-mem (black) or Hb9-mem (red) promoters. Right: Kaplan-Meier survival curve of memKO lines crossed with Prion-mem Tg (Prion-mem, black) or Hb9-mem Tg (Hb9-mem, red) lines. (D) Membralin expression in WT, mem-KO, and mem-KO/Prion-mem Tg spinal cord was examined by immunoblot. (E) Schematic of mouse Cre-driver lines crossed with mem $^{\mathrm{fl} / \mathrm{fl}}$ lines to generate cell-specific membralin deletion strains. (F) Survival curves of Cre lines crossed with mem ${ }^{\mathrm{fl} / \mathrm{fl}}$. GFAP-Cre (red) resulted in early postnatal lethality. (C) Sprawling motor reflex with tail suspension in WT $\left(\mathrm{mem}^{\mathrm{fl} / \mathrm{f}}\right)$, or mem ${ }^{\mathrm{fl} / \mathrm{fl}}$ $\times$ GFAP-Cre lines (Astro-mem-KO) at P15. Astrocytic Cre expression in Astro-mem-KO animals was determined by Cre (brown) and GFAP (purple) staining in spinal cord. Scale bars: $60 \mu \mathrm{m}$. (H) Histological examination of motor cortex from P2O WT or Astro-mem-KO lines stained for the components indicated. Scale bars: $50 \mu \mathrm{m}$. (I) IBA1, GFAP, or CD68 staining area was quantified ( $n=3$ /genotype, $\mathbf{W T}=1.0$ ). Significance in $\mathbf{A}, \mathbf{B}$, and I was calculated by Student's $t$ test. ${ }^{*} P<0.05,{ }^{* *} P<0.01,{ }^{* *} P<0.001$.

EAAT2 levels are perturbed in ALS remains unclear, several pathways have been previously implicated to modulate EAAT2 levels. TNF- $\alpha$ and downstream NFאB signaling for example have been shown to suppress EAAT2 expression (20-22). While these results demonstrate a role for EAAT2 impairment and dysregulated glutamate homeostasis in ALS, mechanisms underlying pathological EAAT2 dysregulation and whether altered pathological TNF- $\alpha$ / $\mathrm{NF \kappa B}$ signaling is involved are currently unclear.

Membralin (Tmem259 or C19orf6) is an evolutionarily conserved ER membrane protein which lacks a well-defined function. Previously, we identified membralin as a novel component of the ER-associated degradation (ERAD) machinery, which reduces $A \beta$ generation by limiting hyperactivation of the $\gamma$-secretase complex (23). However, whether membralin can mediate other neurological functions is unknown. As a physiological role for membralin has not been established, we characterized the effects of membralin on whole-body or central nervous system (CNS) cell type-specific deletion in mice. Whole-body or astrocytic membralin deletion results in loss of motor neurons in spinal cord, accompanied by motor defects, gliosis, and neonatal lethality. Membralin deletion in astrocytes results in dramatic accumulation of extracellular glutamate, resulting in motor neuron glutamatergic toxicity due to reduced expression of the astrocytic glutamate transporter EAAT2. Transcriptomic analysis in mouse cortex with astrocytic membralin deletion results in perturbation of ALS components, including upregulation of Tnfrsfla and downregulation of Eaat 2 transcripts. Interestingly, membralin deletion suppresses EAAT2 expression through a TNF- $\alpha$ /TNFR1/ $\mathrm{NF} \kappa \mathrm{B}$ pathway. Membralin and EAAT2 levels are reduced in human ALS spinal cord and SOD1-mutant mouse models, and membralin expression can ameliorate pathological and lethality effects in the SOD1 ${ }^{G 93 A}$ ALS mouse model. Together, these results reveal a role for membralin function in EAAT2-mediated glutamate excitotoxicity in ALS, and demonstrate a physiological role for membralin in astrocyte-mediated glutamate homeostasis.

\section{Results}

Astrocyte-specific membralin deletion results in neonatal lethality and is associated with motor defects. Previously, we have identified membralin as a novel ERAD component which mediates the turnover of a key subunit of the $\gamma$-secretase complex, nicastrin (23). Deletion of membralin leads the accumulation of nicastrin, thereby enhancing $\gamma$-secretase complex activity (23). Although these results implicate membralin in $\mathrm{AD}$ pathogenesis, whether cell-specific deletion of membralin can affect normal physiological processes within the CNS remained unclear. We therefore generated a conditional membralin deletion model using the mouse Cre-LoxP system to determine the effects of membralin deletion in various cell types in the CNS (Supplemental Figure 1A; supplemental material available online with this article; https://doi.org/10.1172/JCI127695DS1). Although no phenotype was observed with membralin deletion at PO (Supplemental Figure 1B), homozygous whole-body (ACTB-Cre) or global CNS (Nestin-Cre) membralin deletion resulted in smaller animals (ACTB-Cre; Figure 1A: P3 pups, and Supplemental Figure 1B) showing signs of paresis at P3 (Supplemental Video 1) and lethality at about P5 (Supplemental Figure 1B). Onset of motor impairment in these pups was accompanied by enhanced motor neuron loss, cell death, and gliosis in spinal cord from membralin knockout (mem$\mathrm{KO}$ ) mouse lines (Figure 1B). As expected, no membralin expression was detected in mem-KO lines by reverse transcriptase-polymerase chain reaction (RT-PCR) or immunostaining (Supplemental Figure $1, \mathrm{C}$ and D). Membralin deletion was observed in the brain and spinal cord, but not in other tissues in a Nestin-Cre deletion line (Supplemental Figure 1E), indicating that lethality was derived from membralin deletion in the CNS. Given the degenerative motor neuron phenotypes observed in mem-KO mice, we next determined whether restoration of membralin expression in CNS/motor neurons could rescue these defects by crossing mem-KO lines with membralin $\mathrm{Tg}$ lines under the regulation of Prion (CNS) or $\mathrm{Hb} 9$ (motor neuron) promoters (Figure 1C). Neural membralin reexpression (Prion-mem) in mem-KO lines restored postnatal viability and motor defects; however, reexpression of membralin in motor neurons ( $\mathrm{Hb} 9-\mathrm{mem})$ had little to no effect in ameliorating lethality and paresis in mem-KO lines (Figure 1, C and D), suggesting that membralin expression in motor neurons may not be critical for motor neuron survival. We also confirmed that the Prion-mem $\mathrm{Tg} \times$ mem-KO line resulted in membralin expression in astrocytes, as assayed by immunoblot analysis (Supplemental Figure 1F). The Hb9-mem $\mathrm{Tg} \times$ mem-KO line was assessed by histological staining (Supplemental Figure 1G). We next determined whether selective deletion of membralin in motor neurons (Hb9-Cre), oligodendrocytes (Olig2-Cre), microglia (CX3CR1$\mathrm{Cre}$ ), or astrocytes (GFAP-Cre) phenocopied the effects seen with whole-brain membralin deletion (Figure 1E). Consistent with the lack of phenotypic restoration with motor neuron-specific ( $\mathrm{Hb} 9$ mem) expression in mem-KO lines, we did not find any detectable phenotypic abnormalities with membralin deletion in motor neurons (Hb9-Cre). Although we observed postnatal lethality in Astro-memKO lines (Figure 1F, red curve), no effects on viability were observed with membralin deletion in oligodendrocytes or microglia (Figure $1 \mathrm{~F}$ ), 

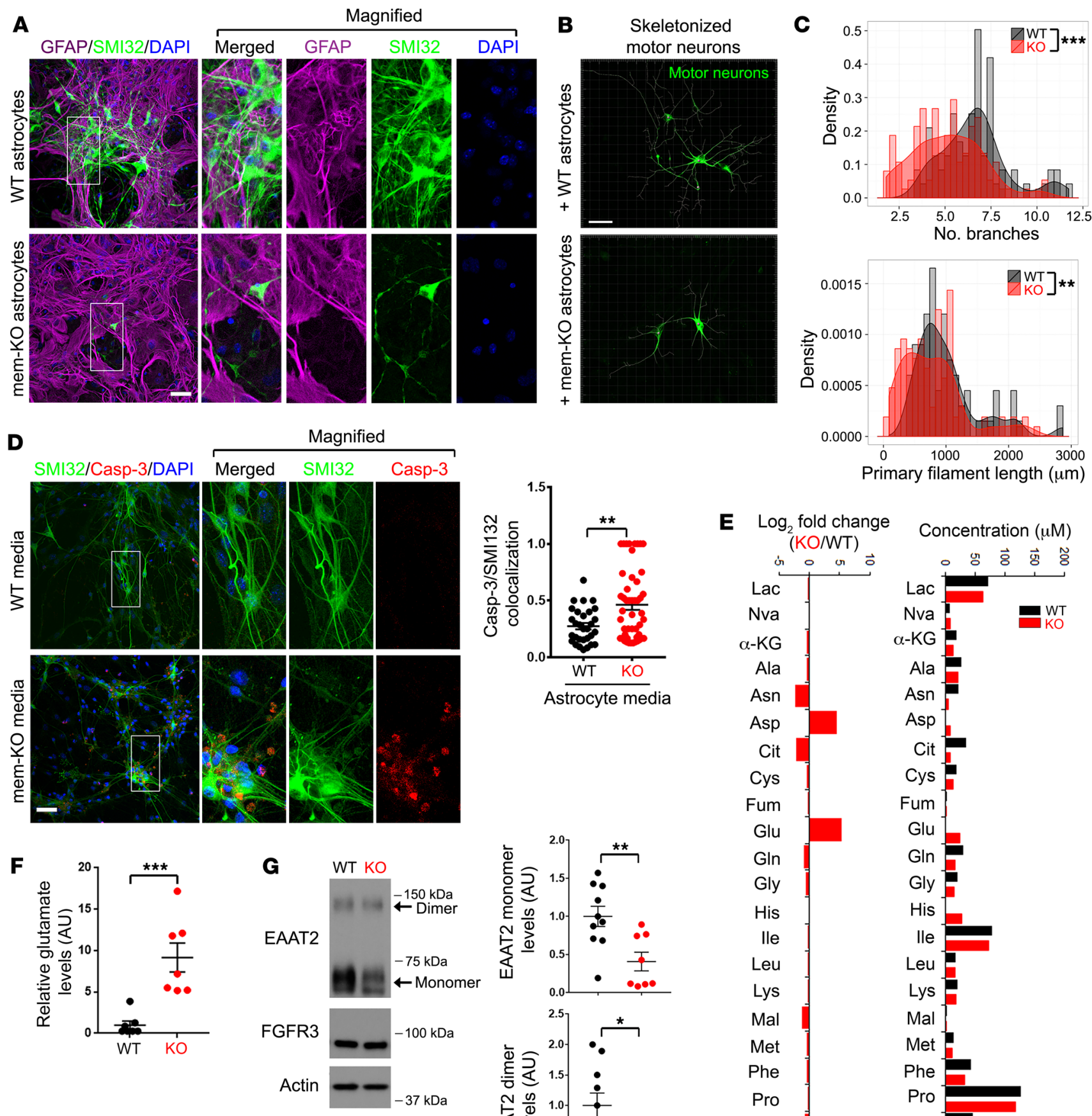

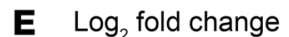

Concentration $(\mu \mathrm{M})$
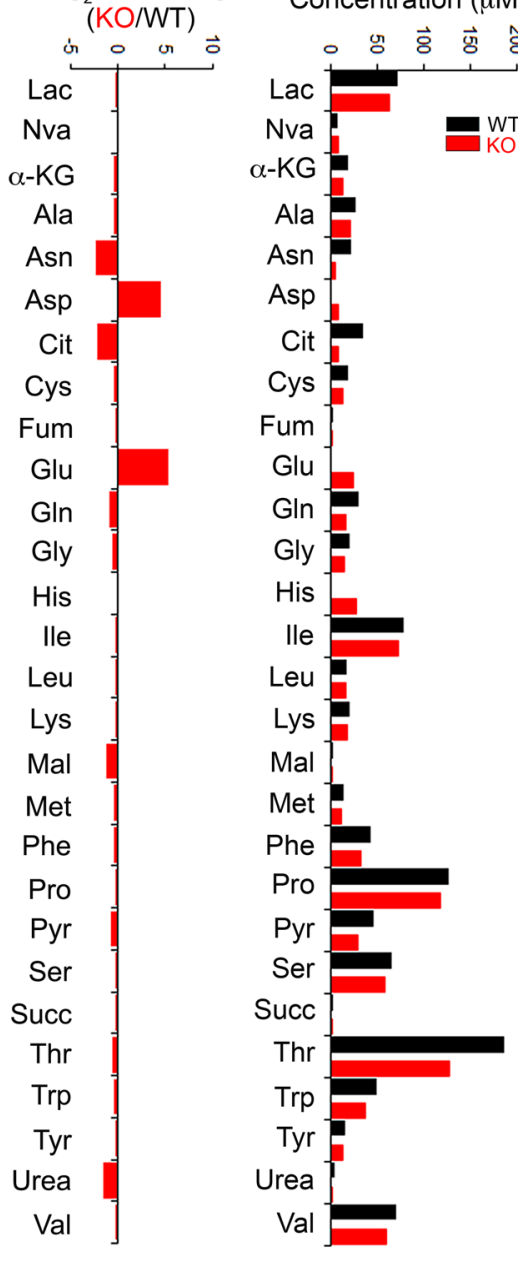
Figure 2. Membralin deletion in astrocytes results in accumulation of neurotoxic components in the conditioned media. (A-C) Mem-KO astrocytes induce motor neuron impairment. (A) WT or mem-KO astrocytes were cocultured with ES-derived (Hb9::GFP) WT motor neurons with SMI32-stained neurites (green), and astrocytes (GFAP, red) were visualized in cocultures. DAPI is indicated in blue. Scale bar: $50 \mu \mathrm{m}$. (B) SMI32-stained neurites in motor neurons from $A$ were skeletonized using Imaris software. (C) Distribution of branch number (upper graph) and primary filament length (lower graph) outputs are presented. (D) WT motor neurons (stained with SMI32, green) derived from mouse ES cells were exposed to conditioned media from WT or mem-KO astrocytes for 24 hours. Caspase 3 activation (red) was visualized for neuronal toxicity. Scale bar: $50 \mu \mathrm{m}$. Quantification depicts SMI32/caspase 3 colocalization normalized to DAPI (mean $\pm \mathrm{SE}, n=30$ for WT and $n=49$ cover glasses for $\mathrm{KO}$ from more than 3 independent experiments). (E) Conditioned media collected from WT (black bars) and mem-KO (red bars) astrocytes was subjected to analysis by GC-MS; absolute values $(50 \mu \mathrm{M})$ (right graph) and $\log _{2}$ (fold change; left graph) in metabolites were characterized over WT $\left(\log _{2}[\mathrm{mem}-\mathrm{KO} / \mathrm{WT}]\right)$. (F) Glutamate in conditioned media from WT ( $n=7$ biological repeats) and mem-KO astrocytes ( $n=7$ biological repeats) was normalized relative to WT samples (set to 1.0, mean $\pm \mathrm{SE}$ ). (C) Lysates from cultured WT (black, $n=10$ biological repeats) and mem-KO (red, $n=8$ biological repeats) astrocytes were subject to immunoblot analysis for the indicated components normalized to actin (WT mean set to 1.0 , mean $\pm \mathrm{SE}$ is shown). Statistical significance values in $\mathbf{C}, \mathbf{D}, \mathbf{F}$, and $\mathbf{G}$ were determined by Student's $t$ test. ${ }^{*} P<0.05,{ }^{*} P<0.01,{ }^{* *} P<0.001$.

indicating that membralin deletion in astrocytes can manifest in deleterious effects potentially related to motor function. As expected, we detected reductions in membralin in GFAP-Cre/mem-KO (Astromem-KO) lines by immunoblot (Supplemental Figure 2A) and histological analysis in spinal cord (Supplemental Figure 2B). No sexual phenotype-specific effects were observed with GFAP-Cre-mediated membralin deletion (Supplemental Figure 2C). We also observed very little change in gliosis and motor neuron numuppber in spinal cord in WT animals compared with mice with membralin deletion in microglia, motor neurons, or oligodendrocytes (Supplemental Figure 2, D and E), indicating that perturbances in motor neuron viability and gliosis with membralin deletion is largely due to the lack of membralin in astrocytes.

Interestingly, membralin deletion in astrocytes incurred a clenching reflex in young mice as opposed to a sprawling phenotype normally seen with tail suspension (Figure 1G). Histological analysis of Astro-mem-KO animals demonstrated prominent gliosis in the motor cortex (Figure $1, \mathrm{H}$ and I), and spinal cord (Supplemental Figure 2F) with a substantially decreased number of motor neurons in spinal cord (Supplemental Figure 2F) suggesting that astrocyte-specific membralin deletion is associated with neurotoxic effects in the CNS. Together, these results indicate that membralin deletion in motor neurons is inconsequential to paresis and postnatal viability, and deletion of membralin in astrocytes manifests motor defects, gliosis, and postnatal lethality. Having previously characterized membralin as a component of the ERAD complex (23), we also determined whether ERAD components were perturbed with membralin deletion in mouse spinal cord (Supplemental Figure 2G). We observed little or no difference in autocrine motility factor receptor (AMFR) or ERAD-associated E3 ubiquitin-protein ligase Hrd1 levels in mem-KO spinal cord (Supplemental Figure 2G).
Membralin deletion in astrocytes induces neuronal toxicity in a non-cell autonomous manner. Since our results indicate that membralin deletion in astrocytes potentially confers neurotoxic effects in a non-cell autonomous manner, we cocultured WT motor neurons with WT or mem-KO astrocytes to observe effects on neuronal viability. Using motor neurons differentiated from mouse Hb9::GFP embryonic stem (ES) cells (Supplemental Figure 3A and ref. 24), astrocytes (WT and mem-KO) cocultured with motor neurons showed no effects on motor neuron viability 1 day after coculture (Supplemental Figure 3B). While we observed little neuronal perturbation with cocultured WT astrocytes, mem-KO astrocytes demonstrated robust effects on perturbing neuritic processes after 7 days of coculture, as revealed by SMI32 staining (Figure 2A). We observed substantially less branch complexity (branching) and a significant impairment in neurite outgrowth in motor neurons cocultured with mem-KO astrocytes (Figure 2, B and C), further indicating that membralin deletion can promote motor neuron impairment. We also observed extensive neurotoxic effects induced in trans by conditioned media from cultured mem-KO astrocytes; conditioned media derived from mem-KO astrocytes incurred deleterious effects revealed by caspase- 3 activation in cultured neurons (Figure 2D). These results indicate that membralin deletion in astrocytes may result in the secretion or accumulation of neurotoxic components in the extracellular environment.

Membralin-KO astrocytes are associated with elevation in extracellular glutamate and reduced EAAT2 expression. To identify potential neurotoxic components in conditioned media from mem-KO astrocytes, we profiled extracellular metabolites in WT and memKO-astrocyte conditioned media by gas chromatography/mass spectrometry (Figure 2E). In comparing secreted components in conditioned media from WT and mem-KO astrocytes, we observed robust elevations in aspartate (23-fold, 4.5- $\log _{2}$ fold increase) and glutamate (42-fold or 5.4- $\log _{2}$ fold increase; Figure $2 \mathrm{E}$ ). Given the role of extracellular glutamate in neuronal excitotoxicity, we further characterized glutamate concentration in mem-KO astrocytes. Indeed, we observed elevations in glutamate in conditioned media from mem-KO astrocytes compared with WT astrocytes (Figure $2 \mathrm{~F}$ ). As these results indicate that excess glutamate in conditioned media from mem-KO astrocytes may induce glutamatergic motor neuron toxicity, we determined whether NBQX (AMPA receptor antagonist) or D-AP5 (N-methyl-D-aspartic acid [NMDA] receptor antagonist) could reverse toxic effects with exposure to mem-KO astrocyte-conditioned media. Indeed, we find that D-AP5 alone or in combination with NBQX could suppress neurotoxicity with exposure to mem-KO-conditioned media (Supplemental Figure 3, C and D). These results indicate that excess glutamate/glutamatergic excitotoxicity is induced by astroglial membralin deletion.

Astrocytes play an essential role in the homeostatic regulation of extracellular glutamate (25), where the glutamate transporter EAAT2 is the primary mediator of extracellular glutamate uptake (4). In cultured WT and mem-KO astrocytes (Figure 2G) and WT and mem-KO mouse spinal cord (Figure 3A), EAAT2 levels were significantly reduced in mem-KO samples as detected by immunoblotting, with comparatively little change in other astrocytic components, such as fibroblast growth factor receptor 3 (FGFR3; Figure $2 \mathrm{G}$ and Figure $3 \mathrm{~A}$ ), connexin 43 (CX43), and EAAT1 (Figure 3, A 

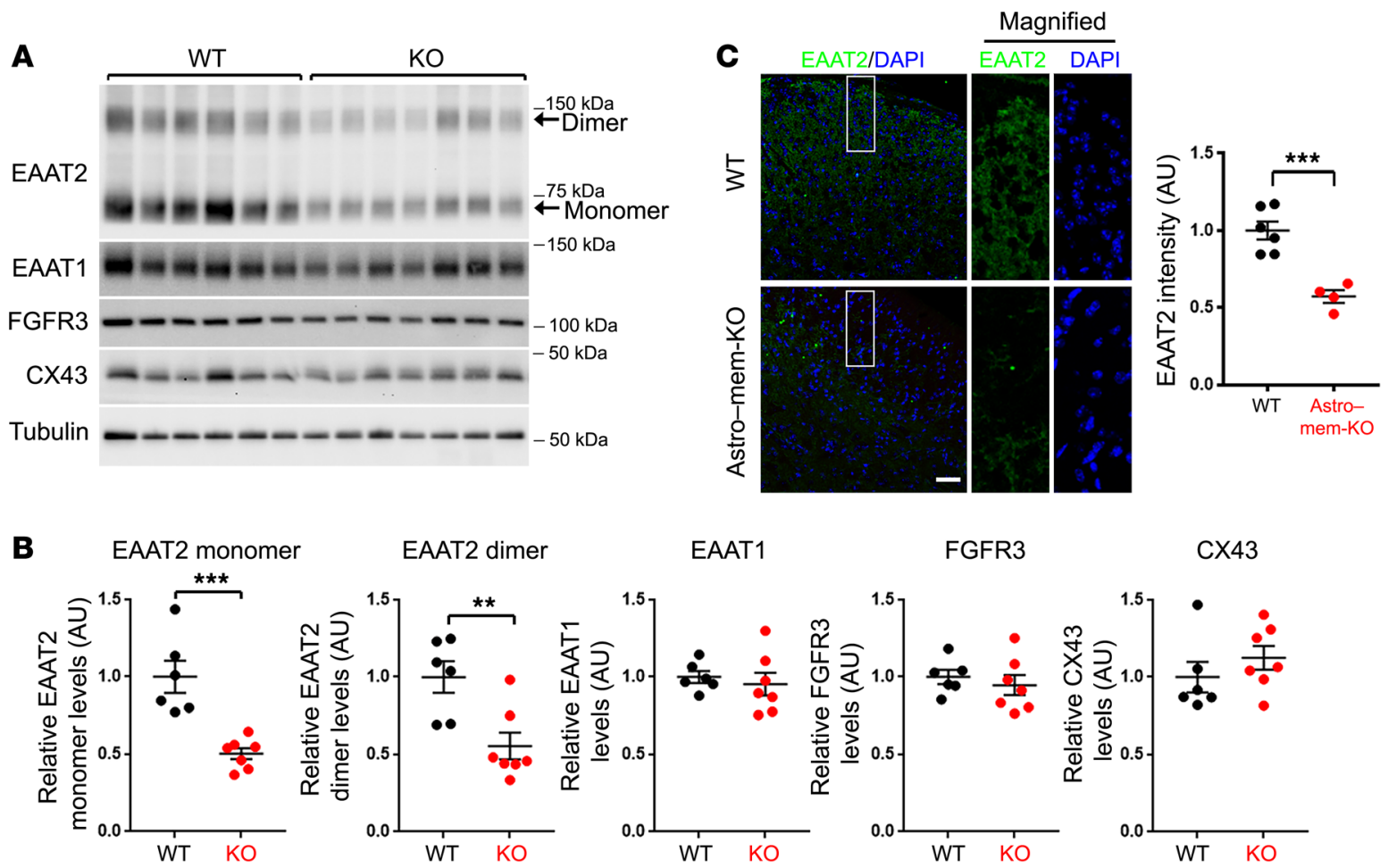

EAAT2 dimer
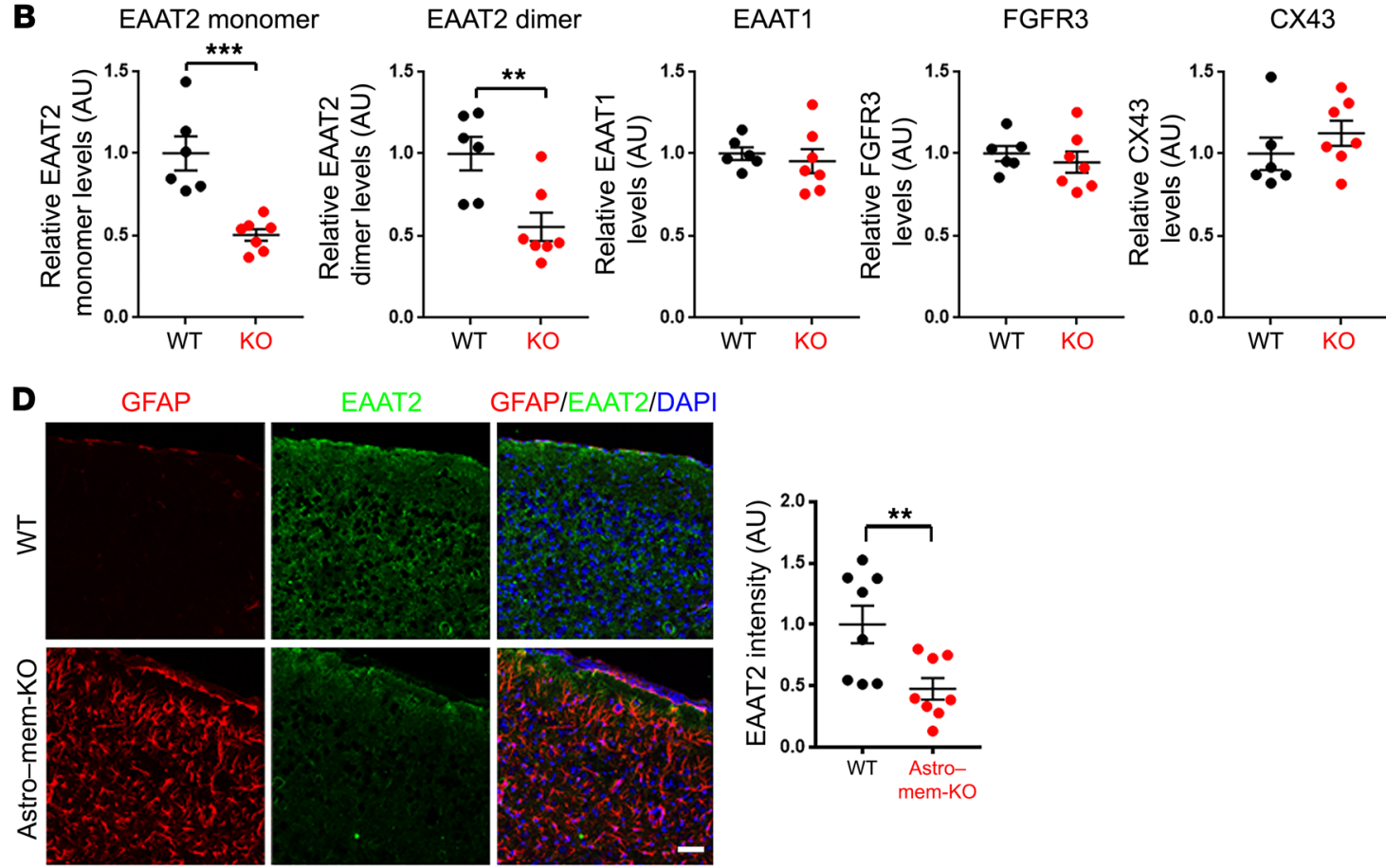

Figure 3. Astroglial membralin deletion results in impaired EAAT2 expression. (A) WT (black, $n=6$ mice) and mem-KO (red, $n=7$ mice) whole spinal cord lysates were analyzed by immunoblot for the components indicated. (B) Quantification of the indicated components are shown. All densitometric values were normalized to tubulin, relative to WT samples (set to 1.0, mean \pm SE). (C-D) EAAT2 depletion in (C) spinal cord and (D) motor cortex of Astro-mem-KO mice was characterized by histological staining. Scale bars: $50 \mu \mathrm{m}$. EAAT2 staining intensity from WT $(n=6)$ and Astro-mem-KO $(n=4)$ spinal cords (C); WT $(n=8)$ and Astro-mem-KO $(n=8)$ motor cortexes (D) were quantified. All graphs in B, C, and D depict mean \pm SE. Significance values were determined by Student's $t$ test. ${ }^{* *} P<0.01,{ }^{* *} P<0.001$

and B). Similarly, histological staining confirmed significant reduction of EAAT2 expression in Astro-mem-KO mouse spinal cord (Figure 3C) and motor cortex (Figure 3D). Together, these results demonstrate that membralin deletion can induce excessive glutamate accumulation and impaired EAAT2 levels in astrocytes.

Normalizing EAAT2 levels in membralin-KO astrocytes eliminates excitotoxic effects in neurons. Thus far, we have established a role for membralin in limiting glutamatergic neurotoxicity, where membralin deletion can reduce astroglial EAAT2 expression. We next determined whether exogenous EAAT2 expression can restore glutamate homeostasis and limit neurotoxic effects associated with membralin deletion. To this end, we used adeno-associated virus (AAV)-EAAT2 constructs expressing EAAT2-HA under the regulation of a GFAP promoter to restore EAAT2 levels in mem-KO astrocytes to WT levels (Figure 4A). AAV-mediated EAAT2 expression in mem-KO astrocytes significantly reduced toxicity induced by mem-KO astrocytes in coculture with murine motor neurons (Figure 4, B and C). Although conditioned media from mem-KO astrocytes conferred significant effects in aggravating caspase-3 activation in mouse motor neurons (Figure 2D), conditioned media from mem-KO astrocytes with AAV-EAAT2 construct significantly reduced caspase-3 activation (Figure 4D). Together, these results indicate that restoration of EAAT2 levels in mem-KO astrocytes reduced non-cell autonomous effects on neuronal toxicity in cultured neurons. 
A



EAAT2

Actin



C
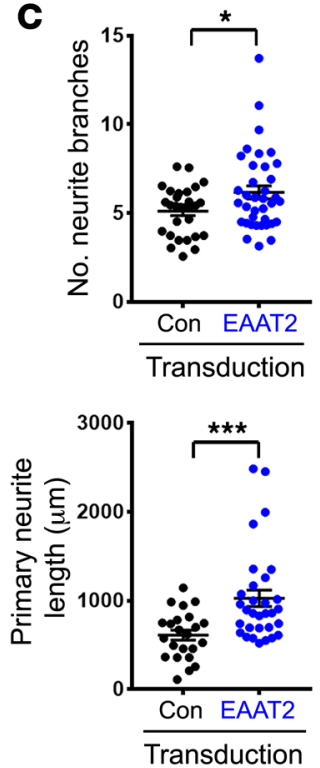

B

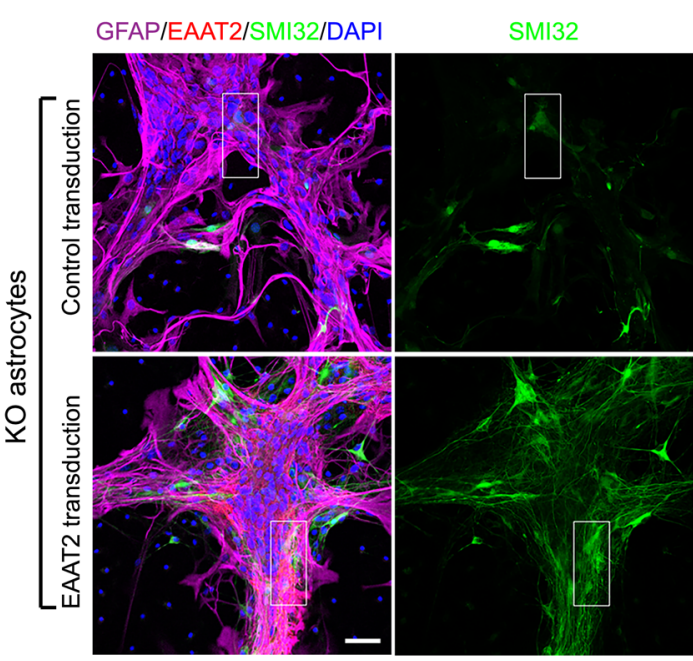

Magnified

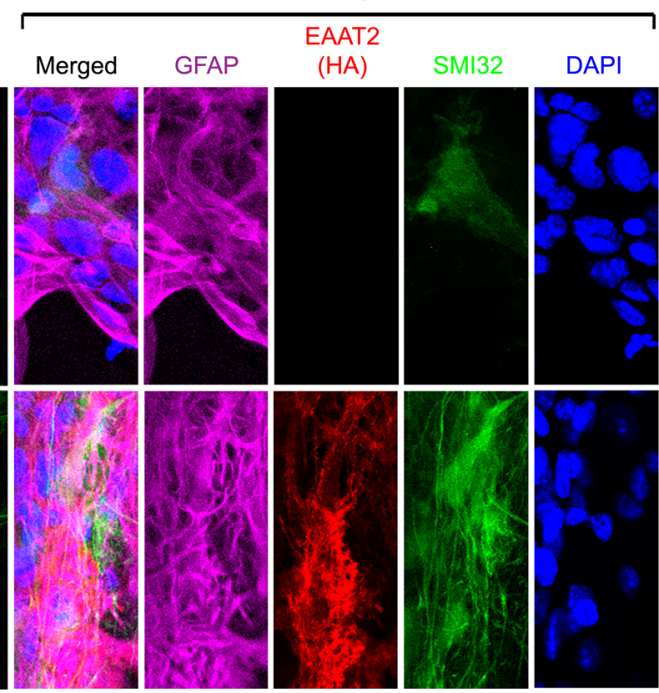

D
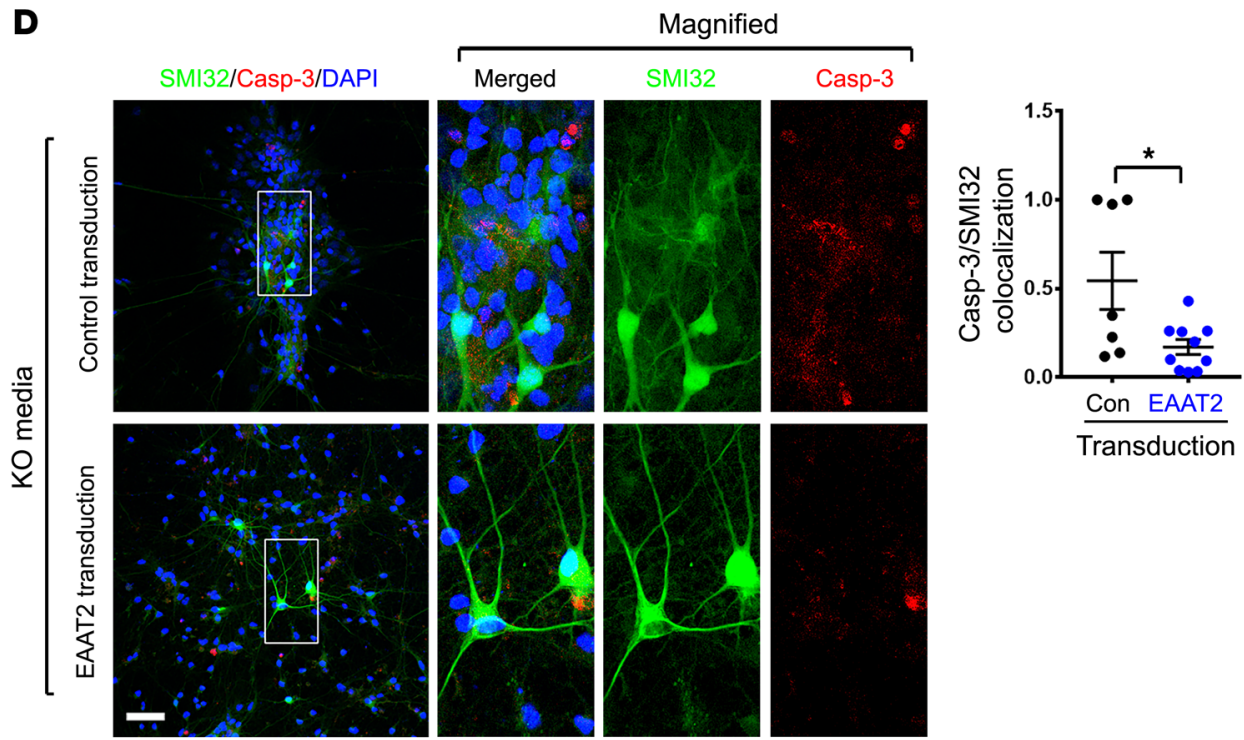

Transduction

Figure 4. Restoration of EAAT2 levels suppresses astroglial neurotoxicity with membralin deletion. (A) Restoring EAAT2 expression levels in memKO astrocytes. Cell lysates from mem-KO astrocytes transduced with control or GFAP-EAAT2-HA AAV vectors or nontransduced WT astrocytes were immunoblotted for EAAT2 or actin as indicated. (B) Murine WT motor neurons were cocultured with mem-KO astrocytes transduced with control or EAAT2-HA AAV constructs for 7 days. Neurons and astrocytes were stained and visualized for GFAP (purple), HA (red), SMI32 (green), and DAPI (blue). Scale bar: $50 \mu \mathrm{m}$. (C) SMI32-stained motor neurons from B were subject to neurite branch quantification for number (left) and length (right). Each dot represents the mean of the motor neurons from individual fields, from $n=4$ independent experiments (mean $\pm S E$ ). (D) Conditioned media from memKO astrocytes transduced with control AAV (upper panels) or EAAT2-HA AAV (lower panels) were applied to mouse motor neurons for 24 hours, where neurons were stained for SMI32 (green), active caspase 3 (red), and DAPI (blue). Scale bar: $50 \mu \mathrm{m}$. Number of active caspase 3-stained motor neurons (normalized to DAPI) were quantified in the adjacent graph (each dot represents the mean value from individual cover glass from $n=4$ independent experiments, mean $\pm \mathrm{SE}$ ). Significance values in $\mathbf{C}$ and $\mathbf{D}$ were determined by Student's $t$ test. ${ }^{*} P<0.05,{ }^{* *} P<0.001$.

Membralin deletion features altered transcriptomic signatures which overlap with mouse ALS models and reactive astrocytes. To characterize global perturbations associated with membralin deletion, we performed transcriptomic RNAseq analysis from murine WT and Astro-mem-KO motor cortex (Figure 5A). Unique expression signatures were observed in Astro-mem-KO motor cortex compared with WT (Figure 5A); KEGG pathway analysis of 2515 differentially expressed genes (DEGs, 1354 downregulated, 1160 upregulated) identified differential expression of ALS components (mmu05014) in Astro-mem-KO motor cortex (Figure 5B and Supplemental Figure 4A). Since membralin deletion resulted in motor neuron deficits and early lethality in mice together with observations that membralin deletion can perturb ALS-associated components, we compared these transcriptomic signatures with other mouse ALS disease models. Interestingly, a subset of 31 DEGs induced by membralin deletion overlapped with 108 DEGs 
A

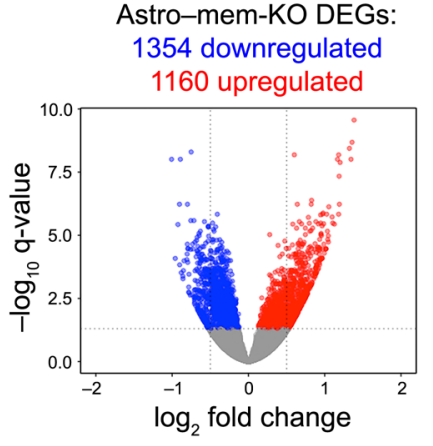

B Astro-mem-KO DEGs, KEGG pathway (mmu05014): ALS $\log _{2}$ fold change

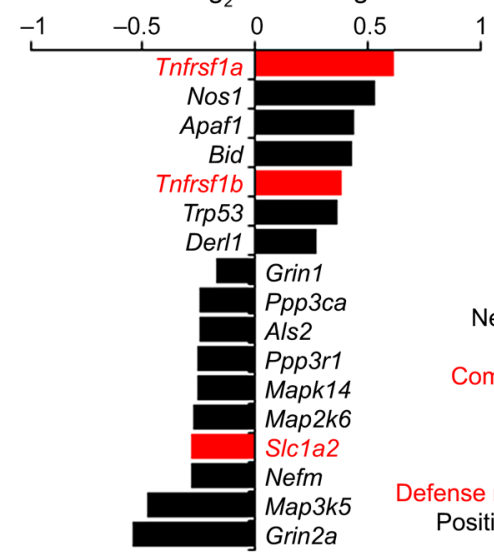

C Astro-mem KO (2515 DEGs)

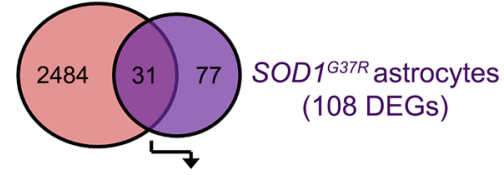

31 overlapping genes

D

No. components $P$ value identified $\left(\times 10^{-1}\right)$ Innate immune response Negative regulation of peptidase activity Immune system process Complement activation, classical pathway Acute-phase response Collagen fibril organization Phagocytosis, engulfment Positive regulation of fibroblast proliferation Epithelial cell differentiation
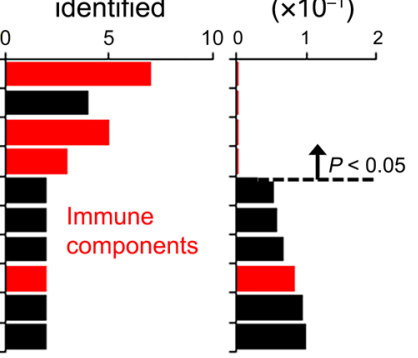



H



Astro-mem KO (2515 DEGs)
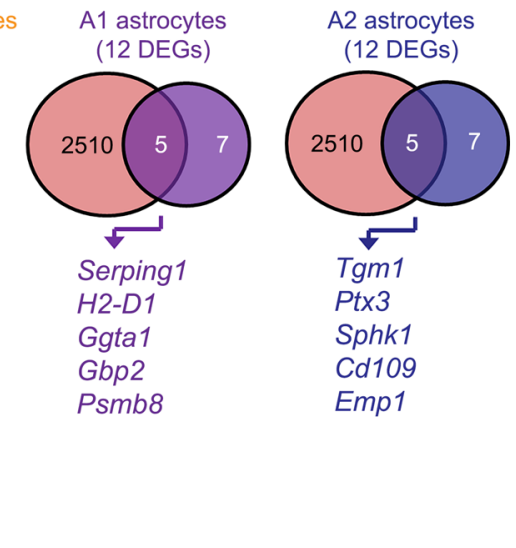

\section{F}

Astro-mem KO (2515 DEGs)

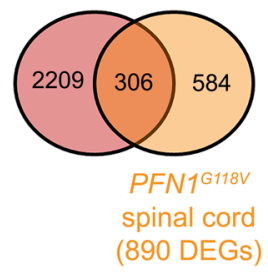

G

Astro-mem KO (2515 DEGs)

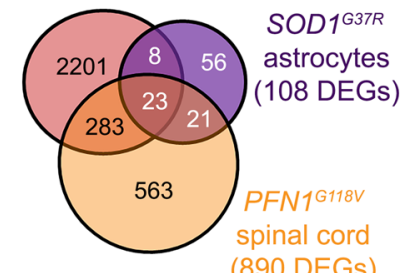



Serpina3n

Vim

Gfap

Serping

Ggta1

Gbp2

Psmb8

Tgm1

Ptx 3

Sphk1

Cd109

Emp1

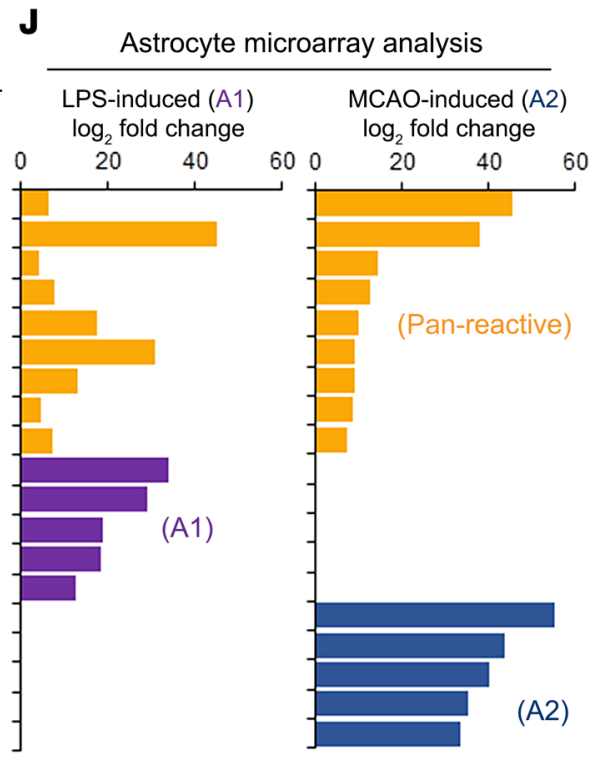


Figure 5. Changes in global transcription with astrocyte membralin deletion overlap with ALS astrocyte and reactive astrocyte signatures. (A) Volcano plot depicting differentially regulated genes (DEGs) identified in Astro-mem-KO and WT mouse motor cortex. (B) Expression of 17 DEGs in the ALS (mmu05014) KEGG pathway category from the 2515 DEGs shown in $\mathbf{A}$ using DAVID Functional Analysis. (C-D) Comparison of Astro-mem-KO

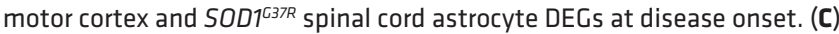
There were 31 overlapping DEGs identified in Astro-mem-KO motor cortex (red) and SOD1C37R astrocytes (purple). (D) Functional CO-BP analysis of the 31 overlapping genes identified in C. Red bars indicate pathways related to immune function. (E) Comparison of differential regulation profiles of Astro-mem-KO spinal cord/SOD1 ${ }^{\text {377R }}$ ALS astrocyte DEGs. The graph on the left shows $\log _{2}$ fold changes in mouse $S O D 1^{637 R}$ astrocytes, the graph on the right shows DEGs identified in Astro-mem-KO motor cortex by RNAseq analysis. Red bars represent immune components in the immune-related $\mathrm{BP}$ categories shown in D. (F) Comparison of Astro-mem-KO and PFN $\mathrm{N}^{\mathrm{C} 118 \mathrm{~V}}$ spinal cord DEGs. There were 306 overlapping DEGs observed in Astromem-KO (red) and PFN ${ }^{G 118 V}$ (dark yellow) spinal cord. (G) There were 23 overlapping DEGs found in Astro-mem-KO (red) and SOD1C37R astrocytes (purple) and $P F N^{C 118 V}$ spinal cord (dark yellow). (H-I) Overlaps of Astromem-KO DEGs with DEGs characterized from A1 neuroinflammatory and A2 ischemia-induced astrocytes. (H) Venn diagrams depicting overlaps of DEGs from Astro-mem-KO spinal cord and pan-reactive (dark yellow), A1 (purple), and A2 (blue) astrocytes. (I) Log fold change of overlapping DEGs shown in $\mathbf{H}$ as characterized by RNAseq analysis. (J) Fold change in expression of overlapping DEGs from $\mathbf{H}$ as characterized previously by microarray in astrocytes treated with LPS or derived from MCAO ischemic models.

observed in mouse SOD1G37R ALS spinal cord astrocytes at onset (Figure 5C and ref. 26). Gene ontology (GO) biological process (BP) analysis of these components included induction of immune function ("innate immune response," "immune system response," and "complement activation, classical pathway;" Figure 5D). Remarkably, fold changes in the 31 overlapping DEGs from Astro-memKO motor cortex and $S O D 1^{G 37 R}$ astrocytes (26) show near-identical expression changes by RNAseq analysis (Figure 5E). Interestingly, transcriptomic changes in Astro-mem-KO motor cortex features significant overlap in DEGs observed in mouse spinal cord in $P F N 1^{G 118 V}$ ALS models (27). In 890 DEGs identified in $P F N 1^{G 118 V}$ spinal cord, 306 DEGs overlapped with astrocytic membralin deletion (Figure 5F). Further, 23 DEGs were found to overlap among Astromem-KO and $P F N^{G 118 V}$ spinal cord and $S O D 1^{G 37 R}$ ALS astrocytes (Figure 5G). Together, this strongly suggests that membralin deletion in astrocytes enacts changes in global expression that overlap with $S O D 1^{G 37 R}$ astrocytes and $P F N 1^{G 118 V}$ spinal cords, including DEG components related to ALS and immune response.

Changes in global expression have also been characterized in murine astrocytes according to the type of astrocyte activation induced by neuroinflammation or ischemia: pan-reactive, proinflammatory neurotoxic A1 activation state, and A2 ischemiainduced activation state (28). Comparing the differentially expressed gene set observed in Astro-mem-KO motor cortex, to pan-reactive, A1, or A2 DEGs (29), we observed overlap between pan-reactive (9 of 13 or $69.2 \%$ of the pan-reactive DEGs); A1 (5 of 12 , or $41.7 \%$ of the A1 DEGs), and A2 DEGs (5 of 12 overlapping A2 DEGs; Figure 5, $\mathrm{H}$ and I). This suggests that membralin deletion in astrocytes potentially induces transcriptional changes associated with reactive astrocytes. More specifically, DEGs upregulated with membralin deletion overlapped with components upregulated with LPS-induced neuroinflammation (A1 reactive astrocytes) and mild carotid artery occlusion (MCAO, A2 astrocytes) previously characterized by microarray (ref. 30 and Figure 5, I and J).

Membralin downregulates EAAT2 expression through a TNFR1/NFKB pathway. Previous studies describe a role for TNF- $\alpha$ in inhibiting glutamate uptake (20), in which TNF- $\alpha$ can suppress EAAT2 expression $(21,22)$. Interestingly, the Eaat2 promoter region comprises NFאB binding sites in both humans and rat (31), where TNF- $\alpha$-dependent NFкB activation is thought to suppress EAAT2 expression $(21,32)$. Our RNAseq analysis identified upregulation of Tnfrsfla, and downregulation of Eaat2 (Slc1a2) within the KEGG ALS pathway in Astromem-KO motor cortex (Figure 5B, indicated in red), indicating that membralin may potentially upregulate TNFR1 to activate $\mathrm{NF \kappa B}$-induced suppression of EAAT2 expression. To test this, we verified that Eaat 2 mRNA levels decreased in Astro-memKO motor cortex samples (Figure 6A) and TNFR1 protein levels increased with membralin deletion (Figure 6B). TNFR1 staining intensity in Astro-mem-KO spinal cord was also elevated compared with WT animals (Supplemental Figure 4B). Further, we observed increased nuclear $\mathrm{NF \kappa B}$ localization in spinal cord from mem-KO animals compared with WT animals (Figure 6C). We also observed increased nuclear NFкB localization in mem-

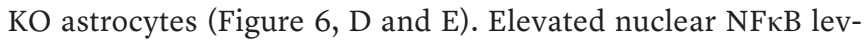
els were reduced with TNFR1 siRNA transfection in mem-KO astrocytes (Figure 6, D and E). Given that EAAT2 levels were alleviated with membralin deletion, we questioned whether suppression of TNFR1 expression could elevate EAAT2 levels in mem-KO astrocytes. Indeed, we observed that TNFR1 siRNA transfection could significantly increase EAAT2 levels (Figure $6 \mathrm{~F})$. Together, these results indicate that membralin deletion suppresses EAAT2 expression through aberrant upregulation of TNFR1/NF $\kappa$ B activity.

Membralin levels are impaired in mouse models of ALS, and correlate with EAAT2 expression in human spinal cord. Previous studies have reported reduced EAAT2 expression in patients with ALS $(14,33)$ and ALS animal models $(17,18)$, suggesting that EAAT2 plays a causal role in inducing glutamatergic neurotoxicity in ALS. It is yet unknown how EAAT2 levels are dysregulated in ALS. Given that astroglial membralin deletion phenocopies motor defects seen in ALS in addition to overlapping changes in global expression observed in $S O D 1^{\text {G37R }}$ spinal cord astrocytes, membralin-dependent effects on EAAT2 expression and glutamate excitotoxicity may also be relevant to ALS pathogenesis. We therefore determined whether membralin levels are perturbed in mouse SOD1-mutant models. Our results indicate that membralin levels in spinal cord tissue from SOD1 ${ }^{G 93 A}$ mouse models of ALS are reduced in the end stage (Supplemental Figure 5A). We also analyzed the association between membralin and EAAT2 levels in $S O D 1^{G 85 R}$ and $S O D 1^{G 37 R}$ mouse models of ALS $(34,35)$. Given that 2 ALS models feature differing rates of onset, we measured EAAT2 and membralin levels at 5-6, 9, and 12 months for $S O D 1^{G 85 R}$ animals, and 4, 12, and 13-15 months in $S O D 1^{G 37 R}$ animals, representing preonset, onset, and end-stage time points of motor dysfunction in these models (Figure 7A). Interestingly, both membralin and EAAT2 levels were reduced during onset and end-stage time points in both models, where a tight correlation between membralin and EAAT2 levels was observed in both $S O D 1^{G 85 R}$ and $S O D 1^{G 37 R}$ animals (Figure 7B). 
A
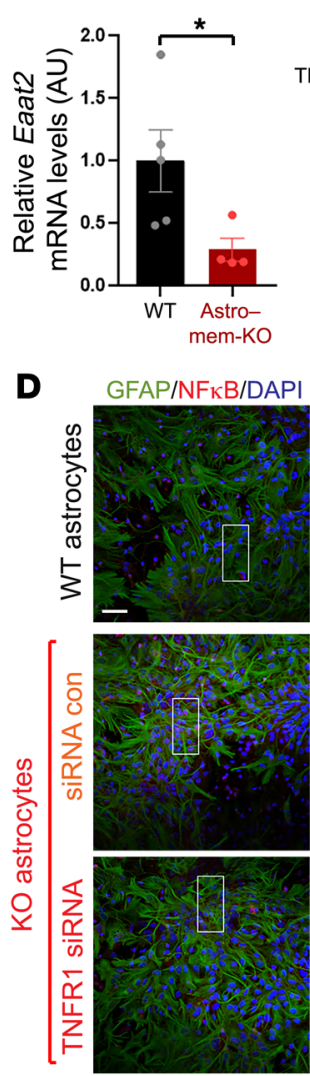
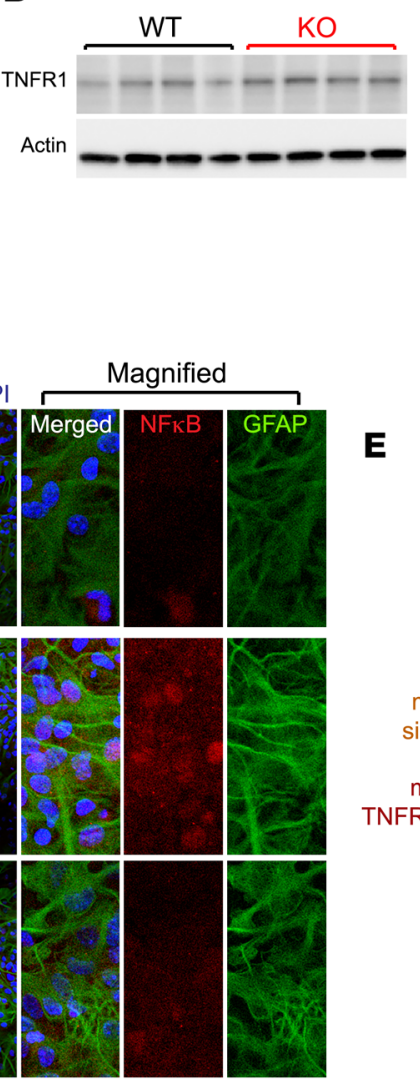



$\mathbf{E}$

$\%$ Nuclear NFKB localization

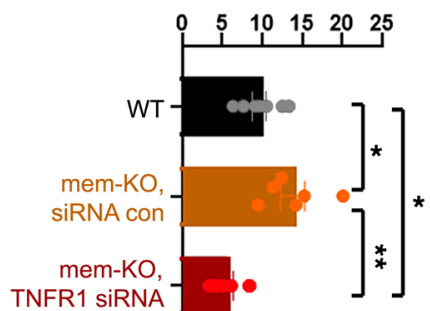

TNFR1 SiRNA
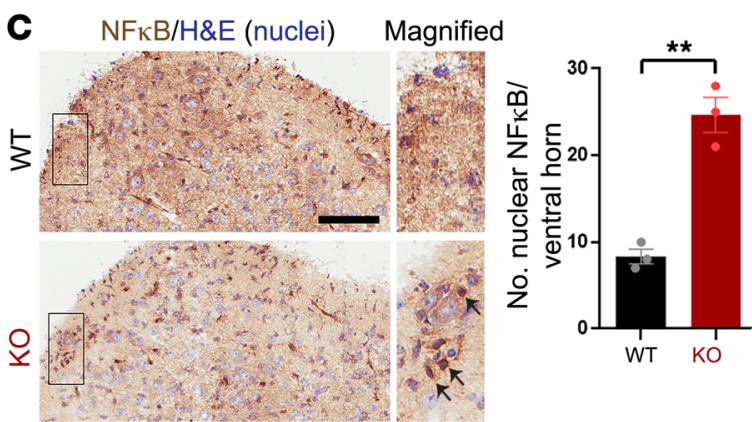
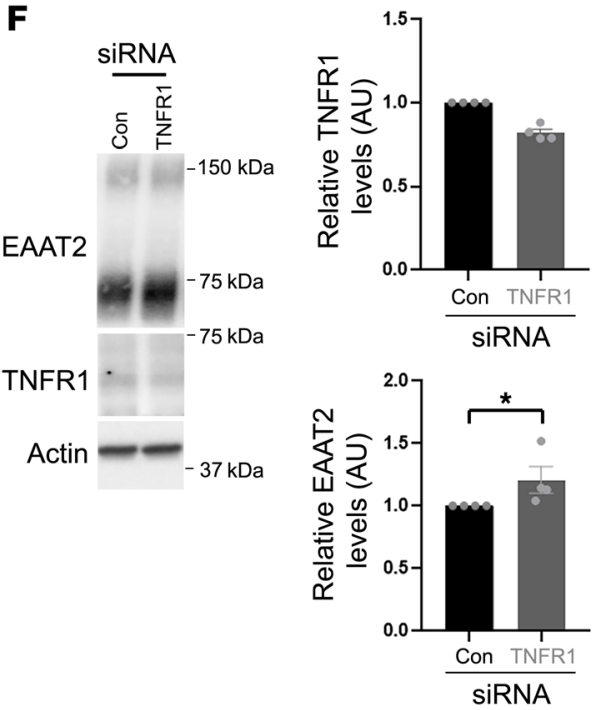

Figure 6. TNFR1/NFKB signaling mediates EAAT2 reductions resulting from membralin deletion. (A) qRT-PCR analysis of Eaat2 mRNA from motor cortex in WT and Astro-mem-KO animals, normalized to 18s rRNA. (B) TNFR1 levels were assessed by immunoblot in spinal cord from WT and mem-K0 animals. Quantification depicts normalized TNFR1 levels (WT mean set to 1.0). (C) Nuclear NFאB localization was examined in P3 WT and mem-KO spinal cord. Number of NFKB-positive nuclei was determined in $n=3$ animals/genotype. Scale bar: $100 \mu \mathrm{m}$. Arrows indicate nuclear NFKB. (D) WT or mem-KO astrocytes were transfected with $50 \mathrm{nM}$ control or TNFR1 siRNA and stained for GFAP (green), NFKB (red), or DAPI. Scale bar: $50 \mu \mathrm{m}$. (E) Quantification of NFKB-positive nuclei shown in D (normalized to DAPI; scale bar: $50 \mu \mathrm{m}$ ). (F) Mem-KO astrocytes transfected with control or TNFR1 siRNA were immunoblotted for EAAT2, TNR1, or actin as indicated. Adjacent graphs depict TNFR1 (left graph) or EAAT2 (right graph) levels. All quantification graphs represent mean \pm SE; statistical significance was determined by unpaired Student's $t$ test (significance in $\mathbf{F}$ was subjected to paired $t$ test analysis), with the exception of $\mathbf{E}$, which was subjected to 1-way ANOVA/Tukey's multiple comparison. ${ }^{*} P<0.05,{ }^{* *} P<0.01$.

Similar to ALS mouse models, we also observed that in spinal cord tissue (cervical region) from patients with ALS where EAAT2 and membralin levels are reduced in familial ALS (fALS) and sporadic ALS (sALS) compared with controls (Figure 7, C and D, and Supplemental Table 2). Importantly, we found that membralin and EAAT2 levels positively correlated in human spinal cord (Figure 7E). Histological analysis in human control and sALS/fALS spinal cord showed more intense membralin and EAAT2 staining in gray matter of control spinal cord, compared with the staining in gray matter of sALS/fALS spinal cord (Figure $7 F$ ). We also evaluated changes in ERAD components (AMFR and Hrd1) in $S O D 1^{G 85 R}$ and $S O D 1^{G 37 R}$ mouse spinal cord and spinal cord tissue from human control, sALS, and fALS patients. We found that ERAD components showed little change in the SOD1-mutant mouse spinal cord (Supplemental Figure 5B), and while some reduction in AMFR levels was observed in human sALS spinal cord (Supplemental Figure 5C), AMFR and Hrd1 levels showed less variability compared with membralin. Together, these results indicated that membralin levels were reduced in ALS mouse models and patients with ALS, and were tightly associated with EAAT2 levels in normal and ALS-associated spinal cord tissue.

Membralin expression in $\mathrm{SOD}^{\mathrm{G}}{ }^{\mathrm{9} 3 \mathrm{~A}}$ astrocytes can reduce non-cell autonomous neurotoxicity in motor neurons. Our results implicated a neuroprotective role for membralin. Given that our results indicated that membralin/EAAT2 expression was attenuated in ALS mouse models and patient samples, we next determined whether elevation of membralin expression in $S O D 1^{193 A}$ murine astrocytes can promote EAAT2 expression and rescue motor neuron impairment. Indeed, AAV-mediated membralin expression in $S O D 1^{693 A}$ astrocytes upregulated decreased EAAT2 levels in $S O D 1^{693 A}$ astrocytes (Figure 7, G-I). In agreement with the notion that membralin may regulate EAAT2 expression through TNFR1/NFkB, membralin expression also reduced TNFR1 levels in $S O D 1^{693 A}$ astrocytes (Figure 7, G and I). Transduction of AAV-Membralin in SOD1 ${ }^{693 A}$ astrocytes also attenuated neuritic defects in cocultured WT motor neurons, compared with control AAV-transduced $S O D 1^{G 93 A}$ astrocyte/motor 
neuron cocultures (Figure 8, A and B). Together, these results indicate that upregulation of membralin levels in SOD1 ${ }^{G 93 A}$ astrocytes can increase EAAT2 levels and rescue $S O D 1^{G 93 A}$ astrocyte-induced motor neuron impairment.

To further investigate whether membralin expression could ameliorate lethality and the pathological effects associated with ALS, we injected SOD ${ }^{\text {G93A }}$ mice at P1 with control or AAV-Membralin viruses into the cerebral-spinal region, and determined effects on survival. We observed that control AAV-injected male mice showed median survival of 157 days, whereas AAV-Membralin-injected male mice had a significantly extended median survival of 170 days in $S O D 1^{G 93 A}$ transgenic animals (Figure $8 \mathrm{C}$ ). Spinal cord tissue from injected animals was processed for histological examination and stained/imaged for EAAT2, GFAP, IBA1, and membralin (Figure 8D). Spinal cord from AAV-Membralin-injected SOD1 ${ }^{G 93 A}$ animals showed significant restoration of EAAT2 staining compared with control AAV/SOD1 ${ }^{\text {G93A }}$ animals (Figure 8, D and E). Membralin expression also reduced gliosis as quantified by GFAP and IBA1 staining in $S O D 1^{G 93 A}$ spinal cord. Together, these results indicated that membralin expression can reverse neurotoxic effects, extend lifespan, and reduce gliosis in the SOD $1^{G 93 A}$ ALS mouse model.

\section{Discussion}

Progressive motor neuron loss in ALS leads to fatality and pathologically manifests selective death of motor neurons within the ventral horn of the spinal cord, brain stem, and motor cortex (36, 37). While familial ALS is derived from mutations in genes such as SOD1 (38), sporadic ALS comprises approximately $90 \%-95 \%$ of all ALS cases in which the cause of motor degeneration remains unclear $(36,37)$. Recent evidence indicates that ALS is a complex disease with a heterologous cell etiology (39) and supports a vital role for astrocytes in aggravating ALS pathogenesis in mouse and iPSC-derived ALS models (40-45).

Aberrant neuronal hyperactivation through excessive glutamatergic stimulation triggers calcium-influx and consequent deleterious events leading to neuronal death $(46,47)$. Glutamate excitotoxicity has been shown to be involved in various neurological disorders, including Parkinson's and Alzheimer's disease and ALS (48-50). Glutamate-dependent excitotoxicity has been proposed to be a primary driver in motor neuron degeneration in both sporadic and familial ALS (5, 10, 14, 15; reviewed in refs. 51-55), indicating that mechanisms involved in extracellular glutamate homeostasis are essential in ALS onset. Although mechanisms underlying pathogenic events in ALS remain elusive, dysregulation of high-affinity glutamate transporters is believed to be etiologically important in degenerative events in ALS (47). EAAT2 is a primary mediator of glutamate reabsorption and is estimated to clear approximately $90 \%$ of all extracellular glutamate through uptake in the CNS (7). Given that reduced EAAT2 levels have been observed in ALS patient spinal cord and motor cortex $(14,33)$, EAAT2 impairment and glutamatergic neurotoxicity is thought to be a key event in ALS onset. Inhibiting downstream glutamatergic stimulation through AMPA-receptor antagonists (LY-300164) is seen to confer protection from kainate-dependent death in motor neurons in vitro (56). Moreover, patients with ALS treated with Riluzole (a putative NMDA/kainate receptor inhibitor) (57) was observed to increase patient survival by 2 to 3 months (58).
Although a definitive treatment strategy to reverse chronic glutamate excitotoxicity remains elusive, restoration of impaired glutamate uptake mechanisms may have robust, long-term effects that may show prolonged efficacy in protecting/restoring motor function with chronic glutamatergic neurotoxicity.

Here, we establish a role for membralin in maintaining EAAT2 levels and glutamate clearance in astrocytes. Our RNAseq analysis implicates aberrant upregulation of TNF- $\alpha$ signaling (Tnfrsfla) and perturbation of other ALS-related components, including Eaat2 with membralin deletion, and our results indicate that membralin downregulation drives EAAT2 suppression through a TNFR1/ $\mathrm{NF \kappa B}$ pathway (Supplemental Figure 6). Given that restoration of membralin levels in mouse SOD1 ${ }^{G 93 A}$ ALS models ameliorates impaired EAAT2 expression and corrects associated pathological/ motor defects triggered by $S O D 1^{G 93 A}$ astrocytes, pharmacological methods to enhance membralin may have promising effects in enhancing glutamate clearance in ALS and other associated neurodegenerative disorders such as AD. Significantly, our results indicate that restoration of membralin can extend ALS mouse lifespan and suppress pathological effects associated with the disease. Interestingly, glutamate excitotoxicity is likely a pathological driver in AD. Future experiments with larger cohorts will be required to substantiate these effects. $A \beta$ was previously found to inhibit EAAT2 distribution to membranes, thereby inhibiting glutamate clearance in acute hippocampal slices (59). Age-dependent reductions in EAAT2 have also been observed in 3xTg-AD (60) and APP23 mouse models (61) and human AD brain (62). Our previous characterization of membralin indicates that membralin expression is attenuated in $\mathrm{AD}$. Together with other studies that describe $\mathrm{AD}$-associated polymorphisms proximal to the membralin locus (63), it appears that membralin levels are impaired in AD. As our work demonstrates a role for membralin in EAAT2 expression/glutamate clearance in ALS, it will be of future interest to determine whether reductions of membralin in AD can also mediate impaired EAAT2 expression/glutamate excitotoxicity in AD.

In summary, we describe a role for membralin in facilitating astroglial EAAT2 expression and extracellular glutamate homeostasis, where astroglial membralin deletion phenocopies the motor defects and lethality observed in ALS mouse models. As restoration of astroglial membralin levels can consequently normalize EAAT2 expression/motor defects and suppress lethality and/or other pathological effects in mouse ALS models, astroglial membralin may be a good pharmacological target to limit excitotoxicity in ALS and other neurodegenerative disorders.

\section{Methods}

Mice. The membralin ${ }^{\mathrm{fl} / \mathrm{fl}}$ line was generated by inserting loxp sites in the introns preceding exon 2 and following exon 4 by Dongxian Zhang at Sanford Burnham Prebys Medical Discovery Institute (SBP) (The Jackson Laboratory, catalog 016574). Membralin homozygous $\mathrm{KO}$ animals were generated by crossing membralin ${ }^{\mathrm{fl} / \mathrm{fl}}$ animals with $\operatorname{Tg}$ (ACTB-Cre) (The Jackson Laboratory, catalog 003376) to achieve germ line deletion of membralin exons 2 to 4 . Membralin heterozygous $\mathrm{KO}$ animals were crossed to maintain the colony and generate homozygous KO animals (mem-KO). Conditional membralin deletion in various cell types in the CNS (astrocytes, microglia, and motor neurons) was achieved by crossing membralin ${ }^{\mathrm{t} / \mathrm{fl}}$ with $\mathrm{Tg}$ (hGFAP-Cre) 
A

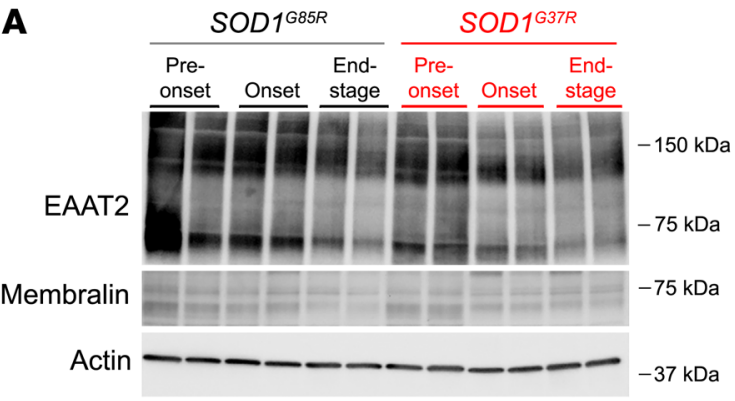

C

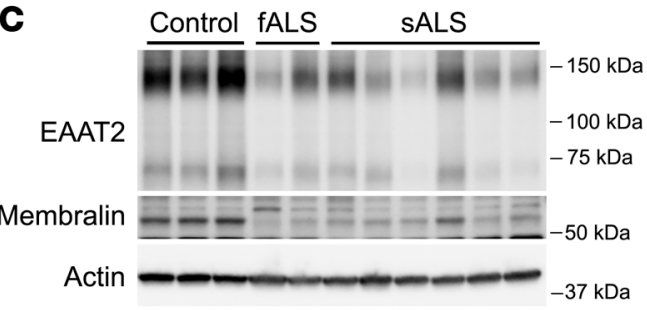

E
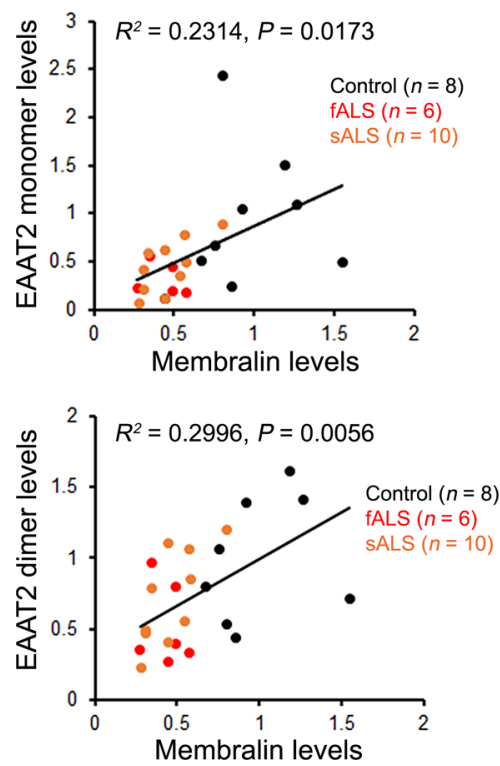

G

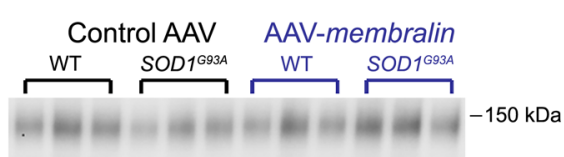

EAAT2

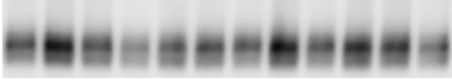

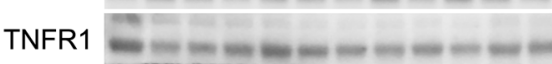
$-75 \mathrm{kDa}$ $-75 \mathrm{kDa}$ Membralin $\quad-\ldots-\cdots--75 \mathrm{kDa}$

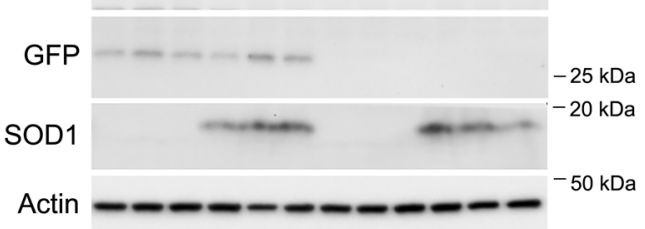

B


D
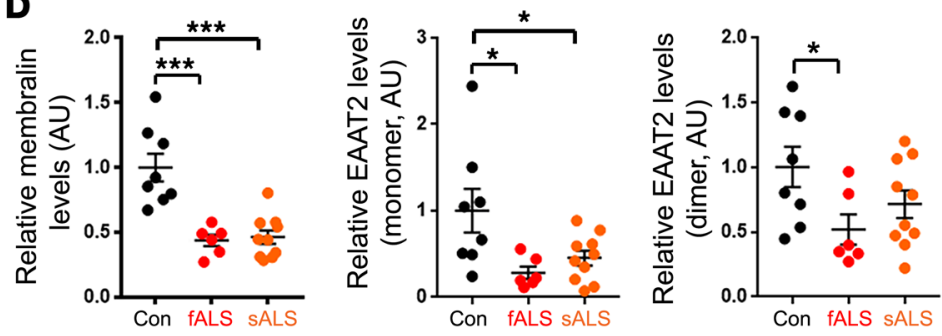

F Membralin
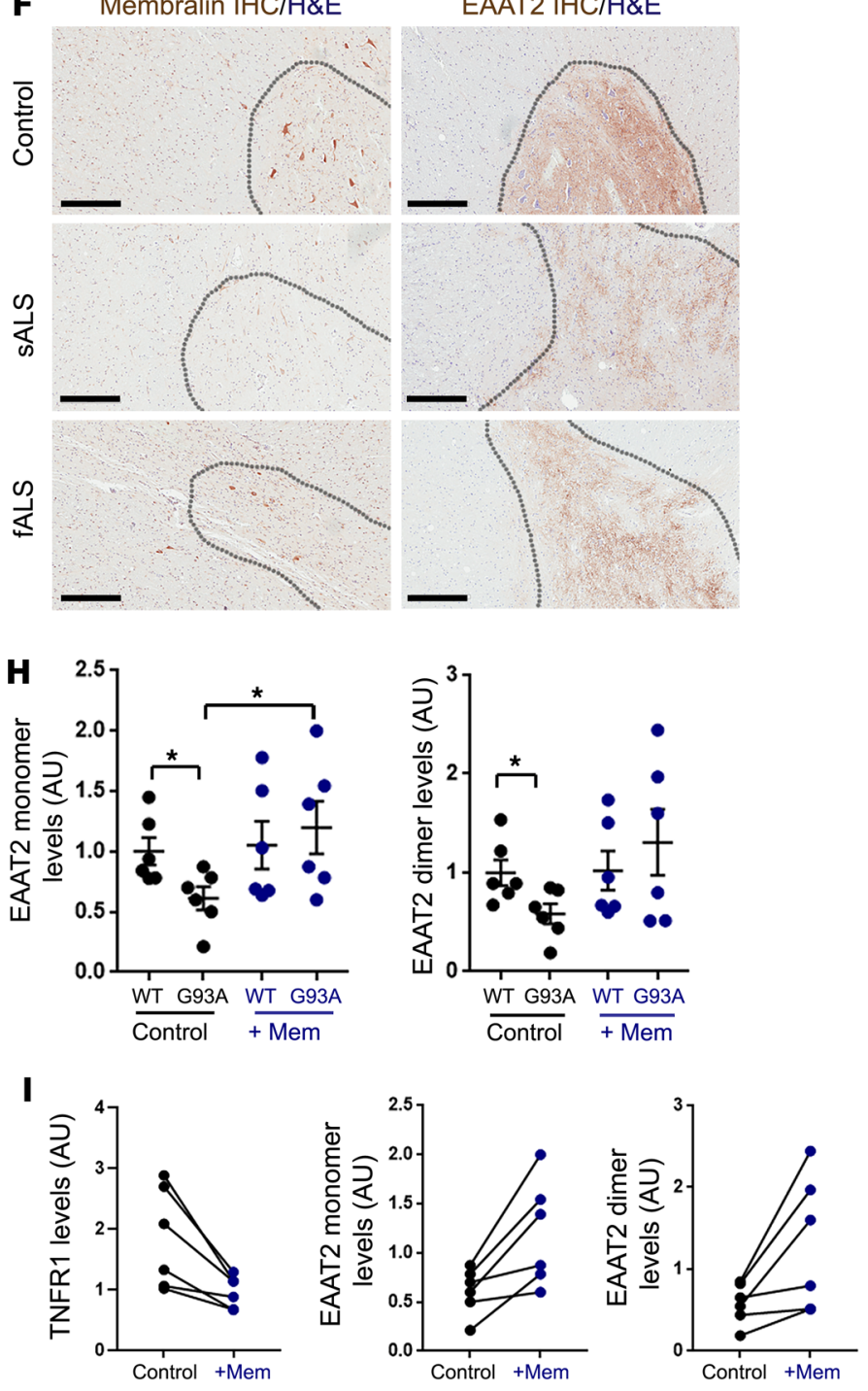

SOD1 ${ }^{\text {G93A }}$ astrocytes 
Figure 7. Reduced membralin levels correlate with reductions in EAAT2 in ALS mouse models and human ALS spinal cord. (A) Lysates from spinal

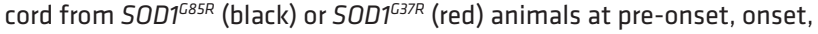
and end-stage time points ( $n=2$ animals) were immunoblotted for EAAT2, membralin, or actin as indicated. (B) EAAT2 monomer (left graph) or dimer (right graph) levels were plotted in comparison to membralin levels in

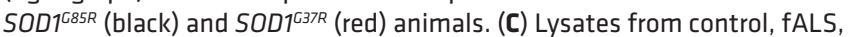
and sALS patient spinal cord samples were immunoblotted for EAAT2 and membralin. (D) Relative membralin and EAAT2 monomer/dimer band intensities were normalized to actin (control sample mean value set to 1.0). Significance values were determined by 1-way ANOVA/Dunnett's multiple comparison. ${ }^{*} P<0.05$, ${ }^{* *} P<0.001$. (E) EAAT2 monomer (upper graph) or dimer (lower graph) levels were plotted in comparison to membralin levels in control (black), fALS (red) or sALS (orange) human spinal cord samples. (F) Human spinal cord samples from control, sALS or fALS were subjected to membralin or EAAT2 staining as indicated. Gray matter regions within the tissues are indicated by dotted gray lines. Scale bar: $300 \mu \mathrm{m}$. (G) Cell lysates from WT ( $n=6$ pups) or SOD1693A ( $n=6$ pups) astrocytes were transduced with control or membralin AAV vectors and immunoblotted for the components indicated. (H) EAAT2 monomer and dimer band intensities in immunoblots from $\mathbf{G}$ were normalized to actin and plotted with respect to WT/AAV control transduced astrocytes (set to 1.0 , mean $\pm S E$ ). Significant values were determined by 1-way ANOVA/ Tukey's multiple comparison. ${ }^{*} P<0.05$. (I) Matched SOD1 ${ }^{\text {C93A }}$ astrocyte cultures transduced with control (black) and membralin (blue) AAV vectors were evaluated for EAAT2 and TNFR1 levels analyzed by paired $t$ tests, showing a significant increase of EAAT2 and decreased TNFR1 levels with membralin transduction relative to controls. $R^{2}$ values (goodness of fit), and 2-tailed significance values for the linear correlation plots shown in B and $\mathbf{E}$ were determined by Pearson correlation analysis.

(a gift from Robert Wechsler-Reya at SBP; The Jackson Laboratory, catalog 004600) for astrocyte-specific cells; Tg (Hb9-Cre) (The Jackson Laboratory, catalog 006600) for motor neuron-specific cells; Tg (Olig2-Cre) (a gift from William Stallcup at SBP, The Jackson Laboratory, catalog 011103) for oligodendrocyte-specific cells; Tg (CX3CR1Cre) (a gift from Marcus Kaul at SBP, The Jackson Laboratory, catalog 025524) for microglia-specific cells; and the Tg (Nestin-Cre) (a gift from Yu Yamaguchi at SBP, The Jackson Laboratory, catalog 003771) line for neural-specific deletion lines. $\operatorname{Tg}\left(S O D 1^{G 93 A}\right)$ was purchased from The Jackson Laboratory (catalog 004435) and crossed with $C 57 B L / 6$ lines. Mice were given ad libitum access to food and water and were maintained in group housing on a 12-hour light-dark cycle.

Antibodies. The following primary antibodies were used in the study: mouse anti-SMI32 (purified anti-neurofilament $\mathrm{H}$ [NF-H], nonphosphorylated) antibody (clone SMI32, 1:500, BioLegend, catalog 801702); mouse anti-GFAP monoclonal antibody (clone G-A-5, 1:500, SigmaAldrich, catalog G6171); goat anti-GFAP polyclonal antibody (1:200, Abcam, catalog ab53554); rabbit anti-IBA1 polyclonal antibody (1:400, Wako, catalog 019-19741); goat anti-IBA1 polyclonal antibody (1:400, Abcam, catalog ab5076); rabbit anti-membralin (mouse) polyclonal antibody (1:1000, customized antibody available from Sigma-Aldrich, catalog ABN1661); rabbit anti-membralin (TMEM259, human) polyclonal antibody (1:1000, Sigma-Aldrich, catalog HPA042669); mouse anti- $\beta$ actin monoclonal antibody (clone AC-15, 1:10,000, Sigma-Aldrich, catalog A5441); rabbit anti-Cre monoclonal antibody (clone D7L7L, 1:100, Cell Signaling Technology, catalog 15036S); rat anti-CD68 (mouse) monoclonal antibody (clone FA-11, 1:300, BioRad, catalog MCA1957GA); rabbit anti-cleaved caspase-3 (Asp175) polyclonal antibody (1:200, Cell Signaling Technology, catalog 9661S); rabbit anti-EAAT2 polyclonal antibody (1:2000, a gift from Jeffery Rothstein at Johns
Hopkins University; ref. 33); rabbit anti-EAAT2 polyclonal antibody (1:100, Cell Signaling Technology, catalog 3838); rabbit anti-EAAT2 (extracellular) polyclonal antibody (1:100, Alomone Labs, catalog AGC022); rabbit anti-FGFR3 monoclonal antibody (1:1000, Abcam, cata$\log$ ab133644); rabbit anti-EAAT1 monoclonal antibody (clone D44E2, 1:1000, Cell Signaling Technology, catalog 5684); rabbit anti-CX43 polyclonal antibody (1:10,000, Sigma-Aldrich, catalog C6219); rabbit anti- $\beta$-Tubulin III polyclonal antibody (1:10,000, Sigma-Aldrich, catalog T2200); rabbit anti-HA-Tag monoclonal antibody (1:500, Cell Signaling Technology, catalog 3724); rabbit anti-TNFR1polyclonal antibody (1:1000, Proteintech, catalog 21574-1-AP); rabbit anti-NFkB monoclonal antibody (clone D14E12, 1:800, Cell Signaling Technology, catalog 8242); rabbit anti-GFP monoclonal antibody (clone D5.1, 1:500, Cell Signaling Technology, catalog 2956); sheep anti-SOD1 polyclonal antibody (1:5000, MilliporeSigma, catalog 574597); mouse anti-GP78-1 (AMFR) monoclonal antibody (clone F-3, 1:500, Santa Cruz Biotechnology, catalog sc-166358); rabbit anti-AMFR (human) polyclonal antibody (1:1000, Cell Signaling Technology, catalog 9590); and rabbit anti-Hrd1 polyclonal antibody (1:1000, Novus Biologicals, catalog NB100-2526).

Virus production and viral transduction. The AAV-GFAP-EAAT2$H A$ plasmid was constructed by subcloning the hEAAT2 cDNA derived from the $C M V-h E A A T 2$ plasmid (a gift from Susan Amara, Addgene plasmid 32814) into an AAV backbone under the regulation of a GFAP promotor (pAAV-GFAP-EGFP, a gift from Bryan Roth, Addgene plasmid 50473), replacing the original GFP fragment. Recombinant AAV vectors were serotyped with AAV9 coat proteins and packaged by Vector Biolabs. AAV-GFAP-EGFP and AAV-GFAPEAAT2-HA were transduced at $10^{10}$ genome copies per milliliter (GC/ $\mathrm{mL}$ ) in astrocyte culture for 7 days prior to coculture with motor neurons or collection of conditioned medium for treatment of motor neurons in trans. The AAV-CAG-membralin plasmid was constructed by subcloning the mouse-Membralin-Myc plasmid $(23,64)$ into an AAV backbone with CAG promotor pAAV-CAG-GFP (a gift from Karel Svoboda, Addgene plasmid 28014). The recombinant AAV vectors were serotyped with AAV9 coat proteins and packaged by Vigene Bioscience. AAV-CAG-GFP and AAV-CAG-Membralin were transduced at $10^{9} \mathrm{GC} / \mathrm{mL}$ in astrocyte culture for 7 days prior to motor neuron coculture experiments.

SOD $1^{G 93 A}$ mouse survival. AAV-CAG-GFP and AAV-CAG-Membralin (at $10^{12} \mathrm{GC} / \mathrm{mL}$ at $2 \mu \mathrm{L}$ volume) were injected into the cerebral-spinal region of the $S O D 1^{G 93 A}$ pups at $\mathrm{P} 1$. The weight of the animals was monitored weekly. The end stage of SOD $1^{G 93 A}$ mouse was determined by its failure to right itself within 30 seconds when placed on its back (65).

Primary astrocyte culture. Primary spinal cord astrocytes were prepared from P1-P3 pups. Spinal cord tissue was treated with papain (20 U/ $\mathrm{mL}$ ) for 20 minutes at $37^{\circ} \mathrm{C}$ in an incubator and centrifuged for 5 minutes at $400 \mathrm{~g}$. Tissue pellets were resuspended in DMEM/F12 media supplemented with $10 \% \mathrm{FBS}$ and $1 \%$ penicillin/streptomycin and mechanically dissociated using $1000-\mu \mathrm{L}$ tips. Cells were seeded and cultured on glass coverslips precoated with Matrigel in 24-well plates for 14-21 days prior to exposure to conditioned medium or motor neuron coculture experiments. To generate conditioned medium $(41,42)$, WT or mem-KO astrocytes were cultured in motor neuron maturation medium (Advance DMEM/F12: AB2 Basal medium [AurnA Biomedical, 1:1], 10\% knockout serum, $1 \%$ penicillin/streptomycin, $1 \%$ L-glutamine, 2-mercaptoethanol, supplemented with GDNF and CTNF). After 7 days, conditioned media was collected. 
A

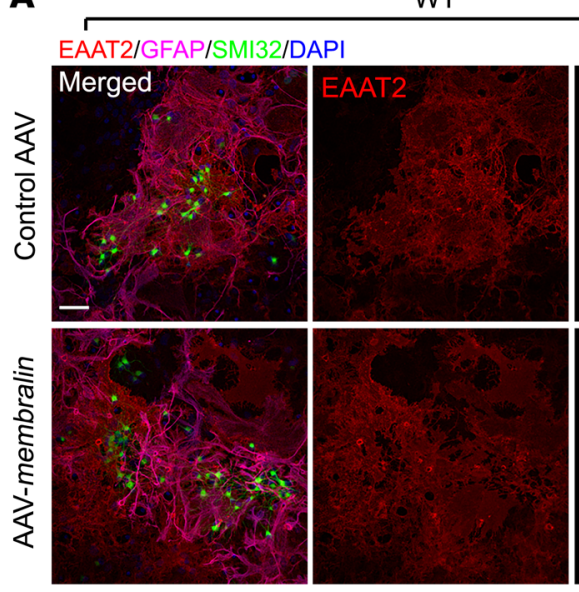

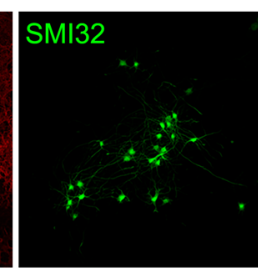

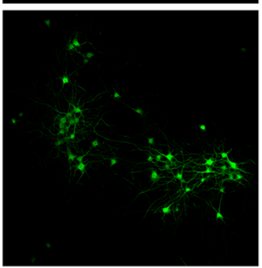

SOD $1^{\mathrm{G93A}}$





D

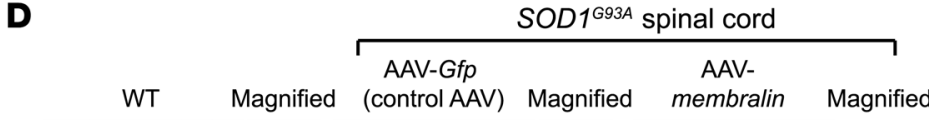

C

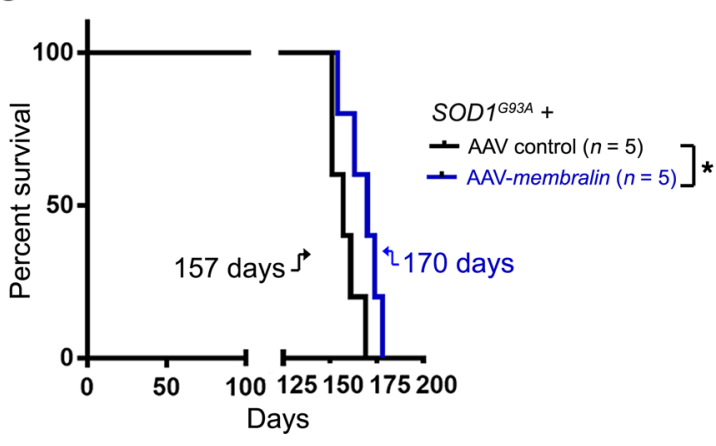

E Relative membralin Relative EAAT2

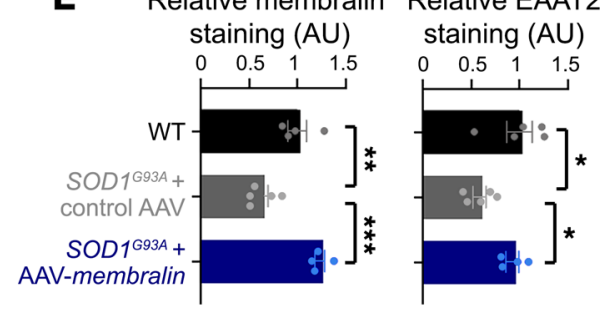

Relative IBA1 Relative GFAP staining (AU) staining (AU)

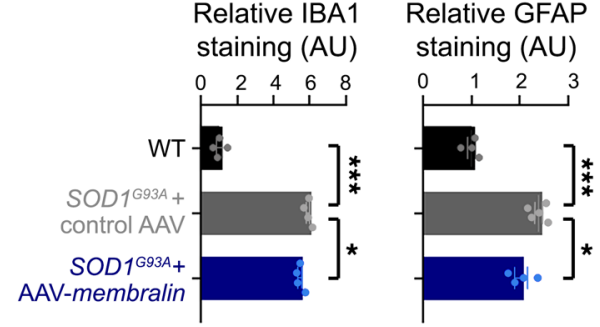


Figure 8. Restoration of membralin levels in an SOD1 ${ }^{693 A}$ ALS model attenuates astrocyte-mediated motor neuron degeneration and extends lifespan. (A) WT or SOD1993A astrocytes transduced with control or membralin AAV vectors were cocultured with WT human motor neurons for 7 days. Cocultures were immunostained for GFAP (purple), EAAT2 (red), SMI32 (green), and DAPI (blue) as indicated. (B) Skeletonized SMI32 images from $\mathbf{A}$ were quantified for neurite length and plotted from at least 3 different views from 8 biological repeats, 2 independent experiments (mean $\pm S E$ ). Significance values were evaluated by 1-way ANOVA/Tukey's multiple comparison. ${ }^{* *} P<0.001$.(C) KaplanMeier survival curves for male $50 D 1^{193 A}$ animals injected with either AAV control (black) or membralin (blue) into the cerebral-spinal region at P1. Median survival is indicated for each genotype. Significance was determined by Mantel-Cox log-rank analysis. ${ }^{*} P<0.05$. (D) Spinal cord tissues from age-matched WT or SOD1 ${ }^{693 A}$ animals injected with control or membralin AAV were stained for EAAT2, GFAP, IBA1, and membralin as indicated. Scale bar: $150 \mu \mathrm{m}$. (E) Quantification of histological analysis on WT (black) and SOD1 ${ }^{C 93 A}$ animals injected with control AAV (gray) and AAV-membralin (blue) are shown (WT values were set to 1.0, mean $\pm S E$ ). Significant differences were evaluated by 1-way ANOVA/Holm-Sidak's multiple comparison. ${ }^{*} P<0.05,{ }^{*} P<0.01,{ }^{* *} P<0.001$

Embryonic stem cell-derived motor neurons. Mouse ESCs (Hb9:: GFP) were purchased from ArunA Biomedical, and differentiated into motor neurons according to specifications provided by the manufacturer. Briefly, ESCs were maintained and grown to $70 \%-80 \%$ confluency in 6-well plates on top of a mouse embryonic fibroblast (MEFs, iXCells Biotechnologies) layer prior to differentiation. To form embryoid bodies (EBs), cells were detached with $1 \mathrm{~mL}$ 0.25\% Trypsin and resuspended in $10 \mathrm{~mL}$ motor neuron differentiation (Advance DMEM/F12: AB2 Basal medium [1:1], 10\% knockout serum, 1\% penicillin/streptomycin, 1\% L-glutamine, and 2-mercaptoethanol). ESCs were plated at $1 \times 10^{6}$ cells per $10 \mathrm{~cm}$ on low-attachment Petri dishes. Day of seeding was defined as differentiation day 0. Medium was changed daily, and cultures were supplemented with retinoic acid (from day 1), purmorphamine (from day 1), and DAPT (from day 4). At differentiation day 6, the EBs were collected and washed in EBSS 3 times prior to dissociation into single cells using Papain $(20 \mathrm{U} / \mathrm{mL}$, Worthington Biochemical Corporation). For conditioned medium experiments, differentiated motor neurons were seeded at $0.1 \times 10^{6}$ cells per PDL/laminin-coated cover glass (Corning). After 7 days of maturation, motor neurons were treated with conditioned medium collected from WT or mem-KO astrocytes for 24 hours, and subjected to immunofluorescence staining. For motor neuron/astrocytes coculture assays $(41,42)$, differentiated motor neurons were seeded at 0.05 $\times 10^{6}$ cells per well in 24-well plates on top of WT or KO astrocyte layers for 7 days and subjected to immunofluorescence staining.

Gas chromatography-mass spectrometry (GC-MS) sample preparation and analysis. GC-MS analysis was performed by methods previously described (66). Culture medium samples $(40 \mu \mathrm{L})$ were mixed with $0.4 \mathrm{~mL}-20^{\circ} \mathrm{C}$ methanol/ water $(1: 1 \mathrm{vol} / \mathrm{vol})$ containing $20 \mu \mathrm{M}$ L-norvaline, followed by addition of $0.225 \mathrm{~mL}$ chloroform, $15 \mathrm{sec}$ onds of vortexing (performed 2 times), and centrifugation at $15,000 \mathrm{~g}$ for 5 minutes at $4^{\circ} \mathrm{C}$. The upper aqueous phase was evaporated under vacuum using a miVac centrifugal evaporator (Genevac). Tubes containing standards were concurrently evaporated. Dried samples and standards were dissolved in $30 \mu \mathrm{L} 10 \mathrm{mg} / \mathrm{mL}$ isobutylhydroxylamine hydrochloride (TCI catalog I0387) in pyridine and incubated for 20 minutes at $80^{\circ} \mathrm{C}$. An equal volume of N-tertbutyldimethylsilyl-N- methyltrifluoroacetamide (MTBSTFA) (Soltec Ventures) was added and incubated further for 60 minutes at $80^{\circ} \mathrm{C}$. After derivatization, samples and standards were analyzed by GC-MS using a Rxi-5ms column $(15 \mathrm{~m} \times 0.25$ i.d. $\times 0.25 \mu \mathrm{m}$, Restek) installed in a Shimadzu QP-2010 Plus GC-MS. The GC-MS was programmed with an injection temperature of $250^{\circ} \mathrm{C}, 1.5 \mu \mathrm{L}$ injection volume, and split ratio of $1 / 10$. The GC oven temperature was initially $130^{\circ} \mathrm{C}$ for 4 minutes, rising to $250^{\circ} \mathrm{C}$ at $6^{\circ} \mathrm{C} /$ minute, and to $280^{\circ} \mathrm{C}$ at $60^{\circ} \mathrm{C} /$ minute with a final hold at this temperature for 2 minutes. GC flow rate with helium carrier gas was $50 \mathrm{~cm} / \mathrm{s}$. The GC-MS interface temperature was $300^{\circ} \mathrm{C}$ and (electron impact) ion source temperature was $200^{\circ} \mathrm{C}$, with $70 \mathrm{eV}$ ionization voltage. Standards were run in parallel with the samples. Standard curves were constructed using MetaQuant (67) and metabolites in the samples were quantified using these settings and corrected for recovery of the norvaline internal standard.

siRNA assay. TNFR1 siRNA (catalog 13369) and control siRNA (catalog 6568) were purchased from Cell Signaling Technology. The siRNAs (50 nM) were transfected into mem-KO astrocytes using Lipofectamine RNAiMAX transfection reagent (Thermo Fisher Scientific, catalog 13778075). The cells were collected after 72 hours transfection for immunofluorescence or immunoblot analysis.

Immunofluorescence. For spinal cord or brain tissues, mice were perfused intracardially with $0.1 \mathrm{M}$ PBS ( $\mathrm{pH}$ 7.2). Spinal cord/brain samples were dissected and fixed in $4 \%$ paraformaldehyde (PFA) in $0.1 \mathrm{M}$ PBS for 24-48 hours. Samples were subsequently transferred to $30 \%$ sucrose solutions in $0.1 \mathrm{M}$ PBS until saturated and subjected to cryosection (10 $\mu \mathrm{m}$ for spinal cord samples and $25 \mu \mathrm{m}$ for brain samples). Frozen sections were incubated in blocking solution (5\% BSA, $0.5 \%$ Triton $\mathrm{X}-100$ in $0.1 \mathrm{M}$ PBS) for 1 hour at room temperature and incubated with primary antibodies in antibody diluent at $4^{\circ} \mathrm{C}$ overnight. The following day, sections were washed with $0.1 \mathrm{M}$ PBS and incubated with fluorophore-conjugated secondary antibodies and DAPI at room temperature for 1 hour. After washing in 0.1M PBS, sections were mounted using Prolong Gold mounting medium. For cells seeded on glass coverslips, cells were fixed in $4 \%$ PFA for 20 minutes and staining was performed as described for the tissue staining. Confocal images were obtained using Zeiss 710 NLO and LSM 880 microscope systems. Quantification of motor neuron neurite length was quantified using the Imaris Software (Bitplane) filament function. Active caspase 3-positive motor neurons were analyzed and quantified using Image J Software.

Immunohistochemistry. Dissected spinal cord tissues were fixed in 4\% PFA for 24-48 hours, and processed for paraffin embedding and sectioning. Transverse spinal cord sections ( $5-\mu \mathrm{m}$ thick) were deparaffinized and hydrated prior to the antigen retrieval process in pH 6.0 antigen retrieval solution (Vector Laboratories). Sections were blocked in $3 \% \mathrm{H}_{2} \mathrm{O}_{2}$ and $10 \%$ normal goat serum (Thermo Fisher Scientific) prior to immunostaining with primary antibodies at room temperature for 1 hour. SignalStain Boost IHC Detection Reagent (Cell Signaling Technology) was used together with secondary antibodies, and positive staining signals were developed using a DAB peroxidase substrate kit (Vector Laboratories). TUNEL staining was performed using the ApopTaq Peroxidase In situ Apoptosis Detection kit (EMD Millipore, catalog S7100) following protocols specified by the manufacturer. Scanned images from stained slides were obtained using the Aperioâ AT2 system (Leica). TUNEL-positive cells and NFאB-positive nuclei were quantified 
using NIH ImageJ software in a blinded manner. EAAT2, membralin, GFAP, and IBA1-positive staining signals were quantified using Leica Aperioâ analysis software in a blinded manner.

Motor neuron analysis/quantification. SMI32-positive motor neurons in the ventral horn region were quantified from 8-10 lumbar spinal cord cryosections per animal. A 100- $\mu \mathrm{m}$ distance was maintained between spinal cord sections.

Immunoblotting. Spinal cord or astrocyte samples were homogenized in cold lysis buffer comprising 1\% NP40,1\% digitonin, $50 \mathrm{mM}$ Tris- $\mathrm{HCl}$ (pH 8.0), and $150 \mathrm{mM} \mathrm{NaCl}$ supplemented with Halt Protease and Phosphatase Inhibitor Cocktails (Thermo Fisher Scientific). Lysates were centrifuged at 20,000 $\mathrm{g}$ for 20 minutes at $4^{\circ} \mathrm{C}$ and supernatants were collected. Protein concentrations were determined by bicinchoninic (BCA) assay, whereby protein samples were subjected to immunoblot analysis. Protein (10-25 $\mu \mathrm{g})$ was subjected to electrophoresis using 4\%-20\% TGX precast protein gels (BioRad), transferred to PVDF membranes, and probed with the indicated primary antibodies. Blots were developed using HRP-conjugated secondary antibodies (Cell Signaling Technology) and ECL reagents. Blots were exposed using ChemiDoc Imager (BioRad) and band densities were quantified using ImageJ.

RNA purification and poly-A RNA sequencing. Total RNA was extracted from P22 mouse motor cortex tissue using the RNA Extraction from Tissue kit (Kurabo, RT-S2). RNA integrity was verified and submitted for RNAseq analysis (Novogene Corporation). Poly-A RNA was isolated using the poly-T oligo-attached magnetic beads, and barcoded libraries were made using the NEBNext Ultra Directional RNA Library Prep Kit for Illumina (New England Biolabs). The library was sequenced on the Illumina NovaSeq6000 System (Illumina).

Bioinformatics analysis and $q R T-P C R$ validation. RNAseq sequence reads were aligned to the Mus musculus genome (mm10) using the STAR aligner (https://code.google.com/p/rna-star/) under default settings. Raw sequence data are available in the NCBI's Gene Expression Omnibus database (GSE 130763). Differential transcript expression was determined using the DESeq2 R package (68). DEGs in mem-KO spinal cord $(Q \leq 0.05$; Supplemental Table 1$)$ that overlapped with $S O D 1^{G 37 R}$ astrocytes (35), $P F N^{G 118 V}$ spinal cord (27), and pan-reactive, A1, A2 astrocytes $(29,30)$ previously characterized were identified. KEGG pathway analysis and GO profiles of DEGs were determined using DAVID (database for annotation, visualization and integrated discovery; ref. 69). cDNA from mouse spinal cord samples were generated from mRNA using the Transcriptor First Strand cDNA synthesis kit (Roche) according to specified protocols from the manufacturer. cDNA (200 ng) was used for qRT-PCR analysis using the Applied Biosystems Powerup SYBR Green Master Mix (Thermo Fisher Scientific) together with the BioRad CFX detection system according to the manufacturer's instructions. Expression values for each target gene were normalized to the internal $18 S$ control. Primer sequences used for qRT-PCR analysis are as follows: Eaat2-F: GGCAGCTGGGGATGTACA; Eaat2-R: ACGCTGGGGAGTTTATTCAAGAAT (70); Membralin-F: CACCTACTCACGCCTCTTCC; Membralin-R: ATG-
TAGGCGAGGACGAAAAG; 18s-F：GATGGGAAGTACAGCCAGG; 18s-R: TTTCTTCAGCCTCTCCAGG.

Quantification and statistical analysis. Detailed information with respect to the number of independent repeats, statistical tests used, and significance ( $P$ values) for each figure panel are specified in the respective figure legends. Data are mean \pm SE. For comparisons between 2 groups, 2-tailed Student's $t$ tests were performed. For multiple group (more than 2 groups) comparisons, 1-way ANOVA tests were performed. A $P$ value less than 0.05 was considered significant. Data values were analyzed and graphed using GraphPad Prism analysis software, Excel, and ggplot2.

Study approval. All animal procedures were conducted with approval and supervision by the SBP Institutional Animal Care and Use Committee. Both male and female mice were used in the study. Postmortem human ALS spinal cord samples were provided by John Ravits from the University of California, San Diego, CA. Written informed consent was obtained from the donors and processed/characterized under the ethical and safety guidelines approved by the SBP IRB and under California and NIH guidelines. All patient information is listed in Supplemental Table 2.

\section{Author contributions}

LLJ, BZ, YZ, TYH, and HX designed the experiments and analyzed the data. LLJ, BZ, YZ, XL, TLiu, JPC, LZ, and TYH performed the experiments. LLJ, WX, TLong, and TYH performed the bioinformatics analysis. MJR, HY, DWC, JR, and SDC contributed key reagents. LLJ, TYH, and HX wrote the manuscript. DZ generated the original loxp-floxed membralin mice. All the authors were involved in discussion during manuscript preparation.

\section{Acknowledgments}

We dedicate this article to the memory of Dr. Dongxian Zhang. We thank Dr. Jeffery Rothstein from Johns Hopkins University for sharing the EAAT2 antibodies. We also thank Drs. Kurt Laha and Shouming Du from Brainxell (Madison, Wisconsin, USA) for sharing the human motor neurons. This work was supported by grants from the NIH (R01 AG061875 and R21 AG059217 to TYH; R01 AG038710, R01 AG044420, R01 NS046673, R01 AG056130, R01 AG056114 to HX, and R01 NS27036 to DWC and SDC) as well as from the Tanz Family Fund (to HX) and the Cure Alzheimer's Fund (to HX). We thank all the members of the Xu lab for critical discussion. We are grateful to have support from the Sanford Burnham Prebys Animal Resources Core, Histology Core, Cancer Metabolism Core, and Genomics Core.

Address correspondence to: Huaxi $\mathrm{Xu}$, Neuroscience Initiative, Sanford Burnham Prebys Medical Discovery Institute, 10901 N. Torrey Pines Road, La Jolla, California 92037, USA. Phone: 858.795.5246 (office), 858.646.3100 ext.3318 (lab); Email: xuh@ sbpdiscovery.org.

\footnotetext{
1. Perry TL, Hansen S, Berry K, Mok C, Lesk D. Free amino acids and related compounds in biopsies of human brain. J Neurochem. 1971;18(3):521-528.

2. Lefauconnier JM, Portemer C, Chatagner F. Free amino acids and related substances in human glial tumours and in fetal brain: comparison with nor-
}

mal adult brain. Brain Res. 1976;117(1):105-113.

3. Kanner BI. Ion-coupled neurotransmitter transport. Curr Opin Cell Biol. 1989;1(4):735-738.

4. Lewerenz J, Maher P. Chronic glutamate toxicity in neurodegenerative diseases-what is the evidence? Front Neurosci. 2015;9:469.
5. Rothstein JD, et al. Knockout of glutamate transporters reveals a major role for astroglial transport in excitotoxicity and clearance of glutamate. Neuron. 1996;16(3):675-686.

6. Kiryk A, et al. Behavioral characterization of GLT1 (+/-) mice as a model of mild glutamatergic 
hyperfunction. Neurotox Res. 2008;13(1):19-30.

7. Tanaka K, et al. Epilepsy and exacerbation of brain injury in mice lacking the glutamate transporter GLT-1. Science. 1997;276(5319):1699-1702.

8. Watase $\mathrm{K}$, et al. Motor discoordination and increased susceptibility to cerebellar injury in GLAST mutant mice. Eur J Neurosci. 1998;10(3):976-988.

9. Carriedo SG, Yin HZ, Weiss JH. Motor neurons are selectively vulnerable to AMPA/kainate receptor-mediated injury in vitro. J Neurosci. 1996;16(13):4069-4079.

10. Rothstein JD, et al. Abnormal excitatory amino acid metabolism in amyotrophic lateral sclerosis. Ann Neurol.1990;28(1):18-25.

11. Robberecht $\mathrm{W}$, Philips T. The changing scene of amyotrophic lateral sclerosis. Nat Rev Neurosci. 2013;14(4):248-264.

12. Fuchs A, et al. Selective mitochondrial $\mathrm{Ca} 2+$ uptake deficit in disease endstage vulnerable motoneurons of the SOD1G93A mouse model of amyotrophic lateral sclerosis. J Physiol (Lond). 2013;591(10):2723-2745.

13. Sasaki S, Komori T, Iwata M. Excitatory amino acid transporter 1 and 2 immunoreactivity in the spinal cord in amyotrophic lateral sclerosis. Acta Neuropathol. 2000;100(2):138-144.

14. Rothstein JD, Martin LJ, Kuncl RW. Decreased glutamate transport by the brain and spinal cord in amyotrophic lateral sclerosis. $\mathrm{N}$ Engl J Med. 1992;326(22):1464-1468.

15. Lin CL, et al. Aberrant RNA processing in a neurodegenerative disease: the cause for absent EAAT2, a glutamate transporter, in amyotrophic lateral sclerosis. Neuron. 1998;20(3):589-602.

16. Bendotti C, et al. Transgenic SOD1 G93A mice develop reduced GLT-1 in spinal cord without alterations in cerebrospinal fluid glutamate levels. J Neurochem. 2001;79(4):737-746.

17. Pardo AC, et al. Loss of the astrocyte glutamate transporter GLT1 modifies disease in SOD1 (G93A) mice. Exp Neurol. 2006;201(1):120-130.

18. Howland DS, et al. Focal loss of the glutamate transporter EAAT2 in a transgenic rat model of SOD1 mutant-mediated amyotrophic lateral sclerosis (ALS). Proc Natl Acad Sci U S A. 2002;99(3):1604-1609.

19. Tong J, et al. Expression of ALS-linked TDP-43 mutant in astrocytes causes non-cell-autonomous motor neuron death in rats. $Е M B O \mathrm{~J}$. 2013;32(13):1917-1926.

20. Fine SM, et al. Tumor necrosis factor alpha inhibits glutamate uptake by primary human astrocytes. Implications for pathogenesis of HIV-1 dementia. JBiol Chem. 1996;271(26):15303-15306.

21. Boycott HE, Wilkinson JA, Boyle JP, Pearson HA, Peers C. Differential involvement of TNF alpha in hypoxic suppression of astrocyte glutamate transporters. Glia. 2008;56(9):998-1004.

22. Su ZZ, et al. Insights into glutamate transport regulation in human astrocytes: cloning of the promoter for excitatory amino acid transporter 2 (EAAT2). Proc Natl Acad Sci U S A. 2003;100(4):1955-1960.

23. Zhu B, et al. ER-associated degradation regulates Alzheimer's amyloid pathology and memory function by modulating $\gamma$-secretase activity. Nat Commun. 2017;8(1):1472.
24. Wichterle H, Lieberam I, Porter JA, Jessell TM. Directed differentiation of embryonic stem cells into motor neurons. Cell. 2002;110(3):385-397.

25. Coulter DA, Eid T. Astrocytic regulation of glutamate homeostasis in epilepsy. Glia. 2012;60(8):1215-1226.

26. Sun S, et al. Translational profiling identifies a cascade of damage initiated in motor neurons and spreading to glia in mutant SOD1-mediated ALS. Proc Natl Acad Sci U S A. 2015;112(50):E6993-E7002.

27. Barham C, Fil D, Byrum SD, Rahmatallah Y, Glazko G, Kiaei M. RNA-seq analysis of spinal cord tissues from hPFN1. Sci Rep. 2018;8(1):13737.

28. Liddelow SA, Barres BA. Reactive astrocytes: production, function, and therapeutic potential. Immunity. 2017;46(6):957-967.

29. Liddelow SA, et al. Neurotoxic reactive astrocytes are induced by activated microglia. Nature. 2017;541(7638):481-487.

30. Zamanian JL, et al. Genomic analysis of reactive astrogliosis. J Neurosci. 2012;32(18):6391-6410.

31. Allritz C, Bette S, Figiel M, Engele J. Comparative structural and functional analysis of the GLT-1/ EAAT-2 promoter from man and rat. J Neurosci Res. 2010;88(6):1234-1241.

32. Sitcheran R, Gupta P, Fisher PB, Baldwin AS. Positive and negative regulation of EAAT2 by NFkappaB: a role for N-myc in TNFalpha-controlled repression. EMBO J. 2005;24(3):510-520.

33. Rothstein JD, Van Kammen M, Levey AI, Martin LJ, Kuncl RW. Selective loss of glial glutamate transporter GLT-1 in amyotrophic lateral sclerosis. Ann Neurol. 1995;38(1):73-84.

34. Bruijn LI, et al. ALS-linked SOD1 mutant G85R mediates damage to astrocytes and promotes rapidly progressive disease with SOD1-containing inclusions. Neuron. 1997;18(2):327-338.

35. Wong PC, et al. An adverse property of a familial ALS-linked SOD1 mutation causes motor neuron disease characterized by vacuolar degeneration of mitochondria. Neuron. 1995;14(6):1105-1116.

36. Wijesekera LC, Leigh PN. Amyotrophic lateral sclerosis. Orphanet J Rare Dis. 2009;4:3.

37. Taylor JP, Brown RH, Cleveland DW. Decoding ALS: from genes to mechanism. Nature. 2016;539(7628):197-206.

38. Rosen DR. Mutations in $\mathrm{Cu} / \mathrm{Zn}$ superoxide dismutase gene are associated with familial amyotrophic lateral sclerosis. Nature. 1993;364(6435):362.

39. San Gil R, Ooi L, Yerbury JJ, Ecroyd H. The heat shock response in neurons and astroglia and its role in neurodegenerative diseases. Mol Neurodegener. 2017;12(1):65.

40. Yamanaka K, et al. Astrocytes as determinants of disease progression in inherited amyotrophic lateral sclerosis. Nat Neurosci. 2008;11(3):251-253.

41. Haidet-Phillips AM, et al. Astrocytes from familial and sporadic ALS patients are toxic to motor neurons. Nat Biotechnol. 2011;29(9):824-828.

42. Di Giorgio FP, Carrasco MA, Siao MC, Maniatis T, Eggan K. Non-cell autonomous effect of glia on motor neurons in an embryonic stem cell-based ALS model. Nat Neurosci. 2007;10(5):608-614.

43. Wang L, Gutmann DH, Roos RP. Astrocyte loss of mutant SOD1 delays ALS disease onset and progression in G85R transgenic mice. Hum Mol
Genet. 2011;20(2):286-293.

44. Marchetto MC, Muotri AR, Mu Y, Smith AM, Cezar GG, Gage FH. Non-cell-autonomous effect of human SOD1 G37R astrocytes on motor neurons derived from human embryonic stem cells. Cell Stem Cell. 2008;3(6):649-657.

45. Nagai M, et al. Astrocytes expressing ALSlinked mutated SOD1 release factors selectively toxic to motor neurons. Nat Neurosci. 2007;10(5):615-622.

46. Arundine M, Tymianski M. Molecular mechanisms of calcium-dependent neurodegeneration in excitotoxicity. Cell Calcium. 2003;34(4-5):325-337.

47. Cleveland DW, Rothstein JD. From Charcot to Lou Gehrig: deciphering selective motor neuron death in ALS. Nat Rev Neurosci. 2001;2(11):806-819.

48. Lipton SA, Rosenberg PA. Excitatory amino acids as a final common pathway for neurologic disorders. N Engl J Med.1994;330(9):613-622.

49. Tapia R, Medina-Ceja L, Peña F. On the relationship between extracellular glutamate, hyperexcitation and neurodegeneration, in vivo. Neurochem Int. 1999;34(1):23-31.

50. Meldrum BS. Glutamate as a neurotransmitter in the brain: review of physiology and pathology. J Nutr. 2000;130(4S Suppl):1007S-1015S.

51. Boillée S, Vande Velde C, Cleveland DW. ALS: a disease of motor neurons and their nonneuronal neighbors. Neuron. 2006;52(1):39-59.

52. Rowland LP, Shneider NA. Amyotrophic lateral sclerosis. N Engl J Med.2001;344(22):1688-1700.

53. Bruijn LI, Miller TM, Cleveland DW. Unraveling the mechanisms involved in motor neuron degeneration in ALS. Annu Rev Neurosci. 2004;27:723-749.

54. Julien JP. Amyotrophic lateral sclerosis. unfolding the toxicity of the misfolded. Cell. 2001;104(4):581-591.

55. Shaw PJ. Molecular and cellular pathways of neurodegeneration in motor neurone disease. $\mathrm{J} \mathrm{Neu-}$ rol Neurosurg Psychiatry. 2005;76(8):1046-1057.

56. Van Den Bosch L, Vandenberghe W, Klaassen H, Van Houtte E, Robberecht W. Ca(2+)-permeable AMPA receptors and selective vulnerability of motor neurons. J Neurol Sci. 2000;180(1-2):29-34.

57. Debono MW, Le Guern J, Canton T, Doble A, Pradier L. Inhibition by riluzole of electrophysiological responses mediated by rat kainate and NMDA receptors expressed in Xenopus oocytes. Eur J Pharmacol. 1993;235(2-3):283-289.

58. Miller RG, Mitchell JD, Moore DH. Riluzole for amyotrophic lateral sclerosis (ALS)/motor neuron disease (MND). Cochrane Database Syst Rev. 2012;(3):CD001447.

59. Scimemi A, Meabon JS, Woltjer RL, Sullivan JM, Diamond JS, Cook DG. Amyloid- $\beta 1-42$ slows clearance of synaptically released glutamate by mislocalizing astrocytic GLT-1. J Neurosci. 2013;33(12):5312-5318.

60. Zumkehr J, et al. Ceftriaxone ameliorates tau pathology and cognitive decline via restoration of glial glutamate transporter in a mouse model of Alzheimer's disease. Neurobiol Aging. 2015;36(7):2260-2271.

61. Schallier A, et al. Region- and age-specific changes in glutamate transport in the A $\beta$ PP23 mouse model for Alzheimer's disease. JAlzheimers Dis. 
2011;24(2):287-300.

62. Li S, Mallory M, Alford M, Tanaka S, Masliah E. Glutamate transporter alterations in Alzheimer disease are possibly associated with abnormal APP expression. J Neuropathol Exp Neurol. 1997;56(8):901-911.

63. Desikan RS, et al. Genetic assessment of ageassociated Alzheimer disease risk: development and validation of a polygenic hazard score. PLoS Med. 2017;14(3):e1002258.

64. Yang B, et al. The critical role of membralin in postnatal motor neuron survival and disease. Elife. 2015;4: 0.7554/eLife.06500.

65. Yamanaka K, et al. Astrocytes as determinants of disease progression in inherited amyotrophic lateral sclerosis. Nat Neurosci. 2008;11(3):251-253.

66. Ratnikov B, Aza-Blanc P, Ronai ZA, Smith JW, Osterman AL, Scott DA. Glutamate and asparagine cataplerosis underlie glutamine addiction in melanoma. Oncotarget. 2015;6(10):7379-7389.

67. Bunk B, et al. MetaQuant: a tool for the automatic quantification of GC/MS-based metabolome data. Bioinformatics. 2006;22(23):2962-2965.

68. Anders S, Huber W. Differential expression analysis for sequence count data. Genome Biol. 2010;11(10):R106.

69. Huang da W, Sherman BT, Lempicki RA. Systematic and integrative analysis of large gene lists using DAVID bioinformatics resources. Nat Protoc. 2009;4(1):44-57.

70. Tian G, et al. Translational control of glial glutamate transporter EAAT2 expression. J Biol Chem. 2007;282(3):1727-1737. 